\title{
Long-term culture-expanded alveolar macrophages restore their full epigenetic identity after transfer in vivo
}

\author{
Sethuraman Subramanian (10,2,3,5, Clara Jana-Lui Busch 1,5 , Kaaweh Molawi',3, Laufey Geirsdottir ${ }^{2}$, \\ Julien Maurizio", Stephanie Vargas Aguilar1,2,3, Hassiba Belahbib², Gregory Gimenez², \\ Ridzky Anis Advent Yuda', Michaela Burkon', Jérémy Favret', Sara Gholamhosseinian Najjar (1)', \\ Bérengère de Laval ${ }^{2}$, Prashanth Kumar Kandalla1, Sandrine Sarrazin ${ }^{1,2}$, Lena Alexopoulou ${ }^{2}{ }^{2}$ and \\ Michael H. Sieweke ${ }^{1,2} \bowtie$
}

\begin{abstract}
Alveolar macrophages (AMs) are lung tissue-resident macrophages that can be expanded in culture, but it is unknown to what extent culture affects their in vivo identity. Here we show that mouse long-term ex vivo expanded AMs (exAMs) maintained a core AM gene expression program, but showed culture adaptations related to adhesion, metabolism and proliferation. Upon transplantation into the lung, exAMs reacquired full transcriptional and epigenetic AM identity, even after several months in culture and could self-maintain long-term in the alveolar niche. Changes in open chromatin regions observed in culture were fully reversible in transplanted exAMs and resulted in a gene expression profile indistinguishable from resident AMs. Our results indicate that long-term proliferation of AMs in culture did not compromise cellular identity in vivo. The robustness of exAM identity provides new opportunities for mechanistic analysis and highlights the therapeutic potential of exAMs.
\end{abstract}

esident macrophages can be found in every tissue of the body, where they fulfill diverse functions in homeostasis, immunity and repair $^{1,2}$. Organ-specific functions and challenges induce functional specialization of macrophages, resulting in highly diverse phenotypes of tissue-resident macrophage populations ${ }^{3,4}$. The potential determinants and stability of macrophage identity have been a subject of intense investigation and debate $\mathrm{e}^{5,6}$. In the lung, various transplantation protocols have shown adaptations of macrophages of different origin to the AM niche ${ }^{4,7,8}$, which has led to the suggestion that the niche has a powerful, possibly dominant role ${ }^{5}$. Conversely, AMs and microglia lose part of their tissue-specific gene expression signature when placed in culture ${ }^{1,9-12}$. This is likely due to the loss of critical environmental signals from the niche in vivo, as well as diverse new chemical and physical stimuli encountered in culture. It has remained unclear, however, whether these adaptations to the cell culture environment are permanent or reversible. In particular, it is unknown whether these adaptations involve irreversible changes in chromatin accessibility or whether core epigenetic identity and the sensitivity to respond to correct environmental cues can be maintained through long periods and multiple rounds of cell division in culture.

Cell culture has had enormous impact on the progress of biological research ranging from cancer to stem cell research ${ }^{13}$. In immunology it has boosted vaccine development, the generation of monoclonal antibodies and cellular therapy and has been essential for many fundamental discoveries. For primary macrophages, however, only a few culture systems are available, including the differentiation from bone-marrow progenitors or monocytes and thioglycolate-elicited peritoneal macrophages, which all involve heterogeneous populations of cells ${ }^{14,15}$. Furthermore, whereas T cells can be generated in large numbers for cancer cellular therapies, the proliferative capacity of macrophages in culture is very limited ${ }^{16,17}$. All common macrophage culture protocols therefore have the drawback to only generate a limited number of cells that are heterogenous and usable only in the short term.

Mouse AMs constitutively express low levels of the antiproliferative transcription factors MafB and cMaf, which enables them to access a network of self-renewal genes that are repressed by these Maf factors in other macrophage populations ${ }^{18,19}$. As a consequence, AMs can expand in culture to large numbers and proliferate for extended periods of time ${ }^{19,20}$. It is therefore of high interest for experimental science and potential cellular therapy applications in cancer immunology, infectious disease and regenerative medicine as to whether macrophage identity can be maintained through long-term proliferation in culture.

Here we have compared the transcriptional and epigenetic identity of mouse AMs in long-term culture before and after re-transplantation into the lung niche environment. We observed that the substantial adaptations of AMs to the culture environment were transient and did not compromise the functional long-term integration into the lung alveolar niche. After transplantation, ex vivo exAMs had a transcriptional and epigenetic signature that was almost indistinguishable from resident AMs that had never transitioned through culture, even after long-term proliferation in culture. This indicated that AMs sustained sensitivity to critical in vivo environmental cues through long periods of culture and represent a unique high-fidelity culture that can shuttle between ex vivo experimental manipulation and in vivo validation. Our observations also

'Center for Regenerative Therapies Dresden (CRTD), Technische Universität Dresden, Dresden, Germany. ${ }^{2}$ Aix-Marseille University, CNRS, INSERM, CIML, Marseille, France. ${ }^{3}$ Max-Delbrück-Centrum für Molekulare Medizin in der Helmholtzgemeinschaft (MDC), Berlin, Germany. ${ }^{4}$ Inovarion, Paris, France.

${ }^{5}$ These authors contributed equally: Sethuraman Subramanian, Clara Jana-Lui Busch.凶e-mail: michael.sieweke@tu-dresden.de 

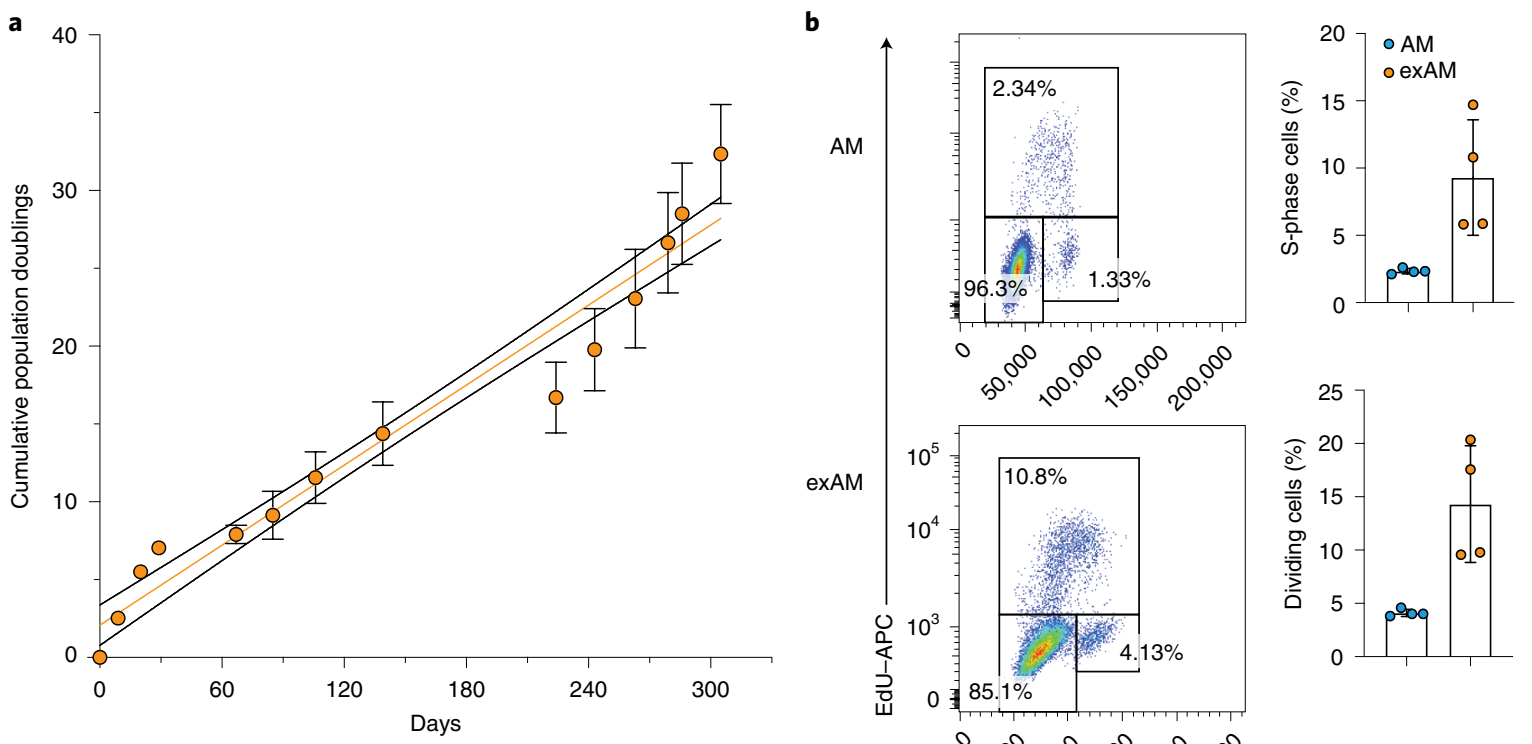

Fig. 1 | Massive ex vivo expansion of AMs in culture. a, Growth curve of AMs in liquid culture represented as cumulative population doublings over time. Linear regression of mean exponential population growth (orange line) with $95 \% \mathrm{Cls}$ (black lines). b, Cell cycle analysis showing percentage of 5-ethynyl2'-deoxyuridine (EdU) incorporation and 4,6-diamidino-2-phenylindole (DAPI) staining in SiglecF+, CD11c ${ }^{+}$AMs and 4 month-cultured exAMs. Dividing cells were calculated as ratio of cells in S-G2M versus G0-G1. Data shown in $\mathbf{a}, \mathbf{b}$ are from biological quadruplicates and represented as mean \pm s.d. and are representative of at least two experiments.

suggest that macrophage expansion ex vivo can provide large-scale preparations for cellular therapy applications with maintained healthy macrophage identity in vivo.

\section{Results}

Expanded AMs maintain phenotype and function in culture. Mouse AMs from bronchoalveolar lavage (BAL) can be cultured ${ }^{20}$ and expanded in granulocyte-macrophage colony-stimulating factor (GM-CSF)-containing medium ${ }^{19}$. We observed that these cells, hereafter referred to as exAMs, could be kept in continuous culture for at least 10 months (Fig. 1a), resulting in 33 theoretical population doublings and an amplification factor of $10^{10}$. This correlated with a three-to-fourfold higher percentage of cells in S phase of the cell cycle in exAMs cultured for 4 months compared to fresh BAL (Fig. $1 b)$, whereas the cell death rate determined by annexin V/7-AAD staining was low (Extended Data Fig. 1a). Furthermore, exAM cultures could also be taken through freeze-thaw cycles without compromising growth capacity (Extended Data Fig. 1b). This suggested an enormous, potentially unlimited expansion potential of exAMs.

Next, we analyzed whether exAM cultures maintained typical macrophage phenotypic and functional characteristics in culture. For practical reasons, all further analyses were performed with 1-4-month exAM cultures. These cells had a typical AM phenotypic appearance and Diff-Quik dye staining properties (Fig. 2a). Furthermore, similar to AMs freshly isolated by BAL, nearly all cells in the exAM cultures had typical macrophage acidified lysosomal structures, indicated by Acridine Orange staining (Fig. 2b and Extended Data Fig. 2a). Nearly all cells also showed labeling with Magic Red dye, a sensor of enzymatic activity of the lysosomal protease cathepsin B (Fig. 2b and Extended Data Fig. 2b) and were also highly active to take up fluorescently labeled latex beads (Fig. $2 \mathrm{c}$ and Extended Data Fig. 2c) as an indicator of phagocytic activity. To further quantify phagocytosis, we used zymosan labeled with the $\mathrm{pH}$-sensitive dye $\mathrm{pHrhodo}$, which only fluoresces upon acidification of the phagocytosed particles in the lysosomes. FACS quanti- fication detected similar fluorescence in exAM cultures and freshly collected AMs, at $37^{\circ} \mathrm{C}$, but not $4{ }^{\circ} \mathrm{C}$ (Fig. $2 \mathrm{~d}$ ), indicating that exAMs maintained full ability to phagocytose pathogen-associated material in an active metabolic process that involved uptake into acidic and enzymatically active lysosomal structures. In addition, stimulation with interferon (IFN)- $\gamma$ and Escherichia coli-derived lipopolysaccharide (LPS), as a TLR4 agonist and a mimetic of infection with a Gram-negative bacterial pathogen, resulted in similar production of reactive oxygen species (ROS) and nitrite in exAM cultures and fresh AMs (Fig. 2e). Furthermore, exAMs were also highly efficient in killing Klebsiella pneumoniae (Fig. 2f), a common cause of bacterial infection of the lung. We further analyzed whether exAMs also maintained a specific AM identity in culture. Flow cytometric analysis indicated that exAMs maintained expression of the specific AM markers SiglecF and CD11c (Fig. 2g), including after a freeze-thaw cycle (Extended Data Fig. 1b). Furthermore, RNA-seq analysis (two pools of three mice each) indicated that the expression of AM-specific core macrophage genes ${ }^{3}$ in exAMs was similar to fresh BAL AMs when compared to other tissue-resident macrophages such as peritoneal macrophages (Fig. 2h). This was reflected in the high gene expression of AM-specific transcription factors ${ }^{3}$, including Pparg, Car4, Cebpb and Bhlhe41 (Fig. 2i), which are key regulators of AM identity and self-renewal ${ }^{4,21-24}$. Cultures of exAMs thus had the central characteristics and functionality of AMs.

Substantial culture adaptations of exAM transcriptome. The microenvironment is a potent determinant of macrophages ${ }^{2,5}$. Because the transfer from the in vivo environment to ex vivo culture results in major gene expression changes in mouse or human $^{10}$ microglia and substantial changes in cytokine responsiveness and glucose metabolism in $\mathrm{AMs}^{11}$, we investigated whether the culture environment also imposed changes in gene expression on exAMs. RNA-seq analysis of exAMs isolated from two pools of three mice each (Extended Data Fig. 3) and cultured for 2 months indicated a substantial number of significant transcriptional changes (false 
discovery rate $(\mathrm{FDR})<0.05)$ compared to AMs in vivo, with 1,370 genes upregulated more than twofold and 376 genes upregulated more than tenfold, whereas 2,317 genes were downregulated more than twofold and 857 genes were downregulated more than tenfold (Fig. 3a). Consistent with the increased number of cycling cells (Fig. 1b), we found enrichment of cell cycle gene sets and increased expression of cell cycle regulators in exAMs compared to AMs in vivo (Fig. $3 \mathrm{~b}$ ). We also found the regulation of transforming growth factor (TGF)- $\beta$-signaling-related genes to be affected (Fig. $3 c$ ), consistent with reports that AM self-renewal depends on an autocrine TGF- $\beta$-signaling loop in vivo ${ }^{25}$. Although exAMs maintained high expression of most core AM-specific genes ${ }^{3}$ (Figs. $2 \mathrm{~h}$ and $3 \mathrm{~d}$ ), expression of specific genes, including genes for surfactant proteins, lipid metabolism and Epcam, a homotypic adhesion molecule, was reduced compared to AMs in vivo (Fig. $3 \mathrm{~d}$ and Extended Data Fig. 4a). This might reflect the loss of the alveolar niche environment or lack of appropriate induction cues in culture.

To analyze the nature of the gene expression changes observed in exAMs in more detail we performed $k$-means clustering. Whereas the majority of genes showed no substantial difference in expression, two clusters of genes were up- and downregulated, respectively, between exAM and AM in vivo (Fig. 3e). Pathway analysis in exAMs compared to AMs in vivo showed upregulation of cytoskeletal, adhesion and migration pathways (Fig. 3f,g), consistent with adaptation to the different surface properties in culture, as well as oxygen response, amino acid and glucose metabolism (Fig. 3f,g and Extended Data Fig. 4b), indicative of altered oxygen pressure and the increased availability of nutrients in culture compared to the alveolar niche, where these parameters are restricted ${ }^{11}$. Pathways downregulated in exAMs compared to AMs in vivo predominantly included immune system pathways, including bacterial and viral response, inflammatory and antigen presentation terms (Fig. 3f,g), consistent with transfer from a barrier tissue constantly exposed to environmental microbes to a sterile tissue culture environment. Together, these data indicated that despite the conservation of core AM identity through long-term culture, a substantial number of genes were up- or downregulated in culture in response to regulatory cues in the changed microenvironment.

exAMs restore transcriptional AM identity in vivo. To determine whether the transcriptional adaptations to the culture environment were stable or reversible, we used intratracheal transplantations to establish whether the original transcriptional identity of AMs could be restored once the microenvironmental cues of the alveolar niche were provided again in vivo. Several protocols have been previously used to transfer macrophages into the alveolar space of the lung and typically involve transplantation into an empty niche, from which resident AMs have been depleted genetically, chemically or by irradiation ${ }^{7,21,26,27}$. Because these procedures all involve strong disturbance of tissue environment and homeostasis, we established a protocol that allowed transplantation into an unmodified niche in wild-type mice. We either transplanted exAMs amplified for 2 months ex vivo (texAMs) or fresh AMs from BAL (tAMs) to control for the effects of transplantation procedure, into nonconditioned wild-type mice (Extended Data Fig. 5a). We detected stable, long-term contribution of the transplanted cells to the AM pool for at least 4 months, with highly similar contribution rates of 5-15\% for both tAMs and texAMs (Fig. 4a). As analyzed by flow cytometry, the expression of the general myeloid and macrophage markers CD11b and CD64 and the AM-specific markers CD11c and SiglecF on tAMs, texAMs and host AMs was indistinguishable 4 months after transplantation (Fig. 4b). These observations indicated that despite extended passage in culture, exAMs acquired a stable AM phenotype and were maintained long-term in vivo.

To further determine whether the global transcriptome could be restored to the in vivo state upon transplantation of exAMs to the in vivo niche, we analyzed tAMs, texAMs and host AMs by RNA-seq analysis (two replicates of pools of three mice each). Besides the high similarity between the two replicates, Spearman's correlation analysis showed a high degree of correlation between texAMs, tAMs and host AMs (Fig. 4c and Extended Data Fig. 3), indicating that the differences between exAMs and in vivo AMs disappeared upon transplantation. Comparison to tissue-resident macrophage populations from published datasets ${ }^{4}$ using principal-component analysis (PCA) indicated that texAMs were indistinguishable from tAMs or resident host AMs, but different to all other resident macrophage populations (Fig. 4d). As an indication of the high degree of their similarity, tAMs, texAMs and host AMs were closer to each other than to the published AM datasets from another laboratory ${ }^{4}$, generated with a different protocol (Fig. 4d).

In fact, only 56 genes were significantly differentially expressed with more than a twofold change (FDR $<0.05)$, between tAMs and texAMs, compared to 3,547 genes that were differentially expressed between exAMs in culture and texAMs (Fig. 4e,f). Similarly, 3,687 genes were differentially expressed between exAMs and host AMs, but only 217 genes between texAMs and host AMs (Fig. 4e). In addition, a similar number of differences as between texAMs and host AMs were also detected between tAMs and host AMs (Extended

Fig. 2 | Expanded AMs maintain phenotype and function in culture. a, Brightfield images and Diff-Quik histological staining on AMs and exAMs (2 months). Scale bars, $20 \mu \mathrm{m}$ and $10 \mu \mathrm{m}$. Images shown are representative of two independent experiments with four technical replicates each. b, Fluorescence imaging of the lysosomal marker Acridine Orange and lysosomal cathepsin B activity using Magic Red on AMs and exAMs (3 months). Further examples are in Extended Data Fig. 2. Quantification showing percentage of lysosome and cathepsin B-activity-positive cells per image, total cells $n=72$ (AMs), $n=71$ (exAMs). c, Fluorescence imaging of phagocytosis of green fluorescent latex beads by AMs and exAMs ( 2 months). Further examples are in Extended Data Fig. 2. Cytoplasm and nuclei stained by ActinRed and DAPI. Scale bars, $5 \mu \mathrm{m}(\mathbf{b}, \mathbf{c})$. d, Flow cytometric analysis showing phagocytosis of pHrhodo Red Zymosan bioparticle conjugates by AMs and 2-month exAM cultures at $4^{\circ} \mathrm{C}$ (control) and $37^{\circ} \mathrm{C}$. e, Photometric quantification of ROS and nitrite production in AMs or exAMs (4 months) stimulated with LPS/IFN- $\gamma$ for $16 \mathrm{~h}$. Data are shown as mean \pm s.d. Nitrite assay: AMs $\star \star P=0.0022$, exAMs ${ }^{\star} P=0.0286$; ROS assay: AMs ${ }^{\star \star} P=0.0040$, exAMs, ${ }^{\star} P=0.0040$ for nontreated (NT) versus LPS/IFN- $\gamma$ stimulated by two-tailed unpaired MannWhitney U-test. f, Bacterial killing assay with K. pneumoniae showing colony-forming units (c.f.u.) per ml in AMs or 4-month exAM lysates at indicated time points after removing bacteria from cells. Data are shown as mean \pm s.d. Data in a-f are representative of at least two independent experiments with at least three replicates each. $\mathbf{g}$, Flow cytometric analysis showing expression of cell-surface markers F4/80, CD64, CD11c, SiglecF and CD11b (colored) against isotype control (gray) on AMs and exAMs (1 month). h. RNA-seq gene expression from two replicates each pooled from three mice/cultures showing core AM upregulated signature genes ${ }^{3}$ in AMs or exAMs ( 2 months) compared to peritoneal macrophages (PMs) and AMs from the Immgen consortium ${ }^{48}$ (GSE122108). The box plot shows the median with the lower and upper quartiles representing a 25th to 75th percentile range with whiskers extending to $1 \times I Q R$ and outliers falling outside of this range. Pairwise comparison between average PMs and other samples by one-sided Wilcoxon signed-rank test Benjamini-Yekutieli adjusted $P$ values $<10^{-18}$. i, Heat map of AM-specific transcription factor gene expression from two replicates each pooled from three mice/cultures of AMs or exAMs (2 months) compared to PMs and AMs (Immgen), showing rlog normalized values from minimum (blue) to maximum (red). 
Data Fig. 5b). Finally, in a tripartite analysis only 24 genes overlapped between the comparisons of texAMs to tAMs and texAMs to host AMs but were not found in the tAM to host AM compari- son (Fig. 4g), of which only nine genes including Slfn5, Msr1 and Itgb3 were regulated in the same direction in texAMs and exAMs. This indicated that the large majority of differences detected a

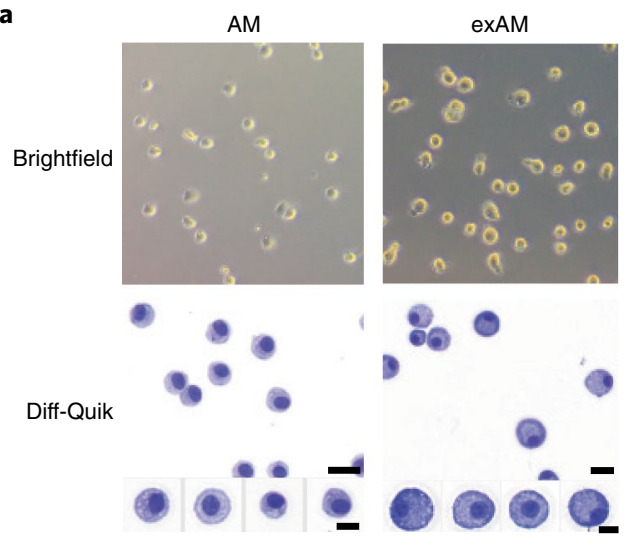

C

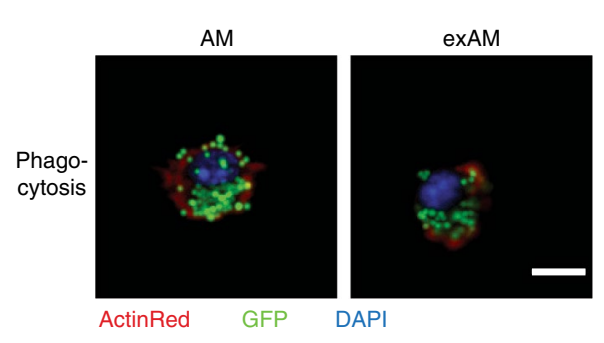

b

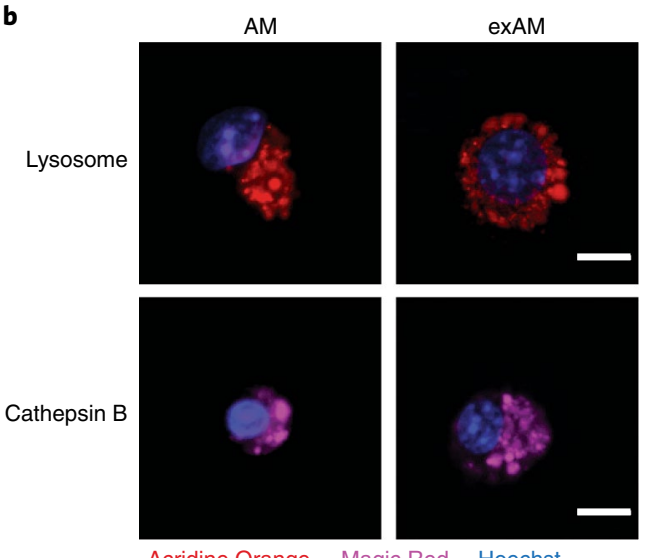

Acridine Orange Magic Red Hoechst

d

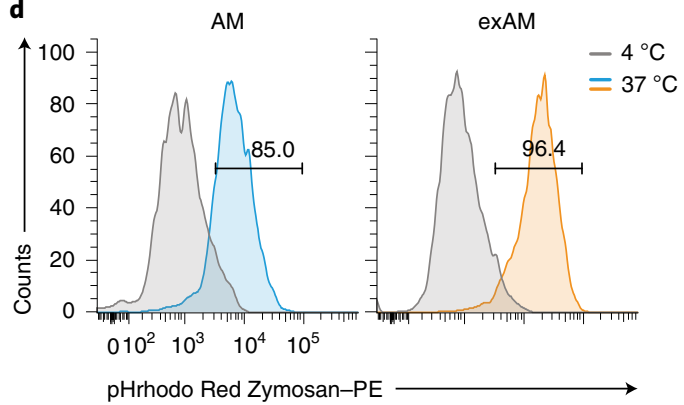

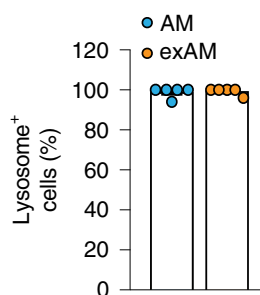
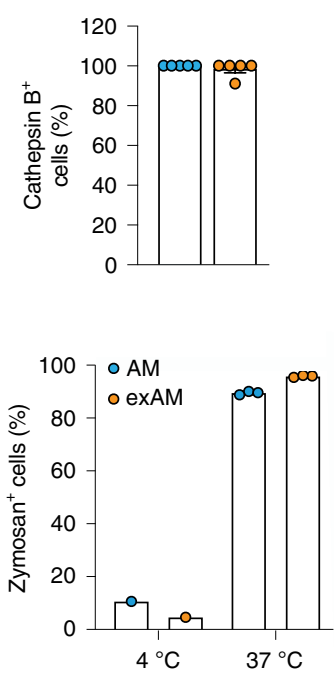

e

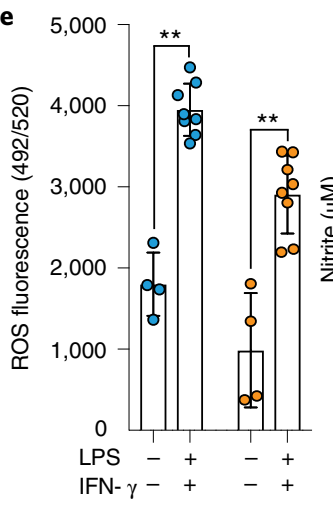

$\mathbf{f}$

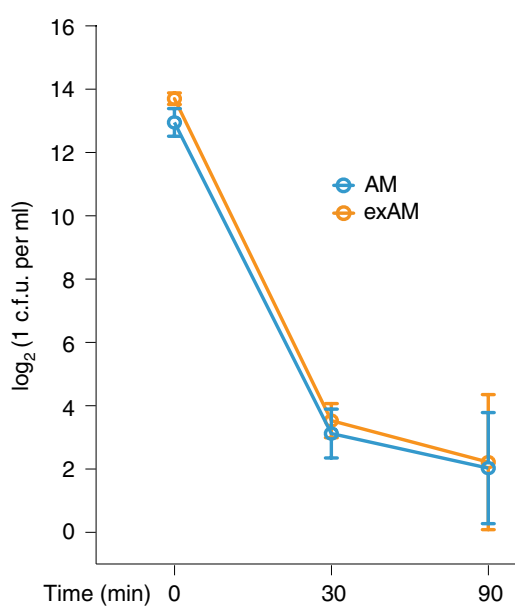

g
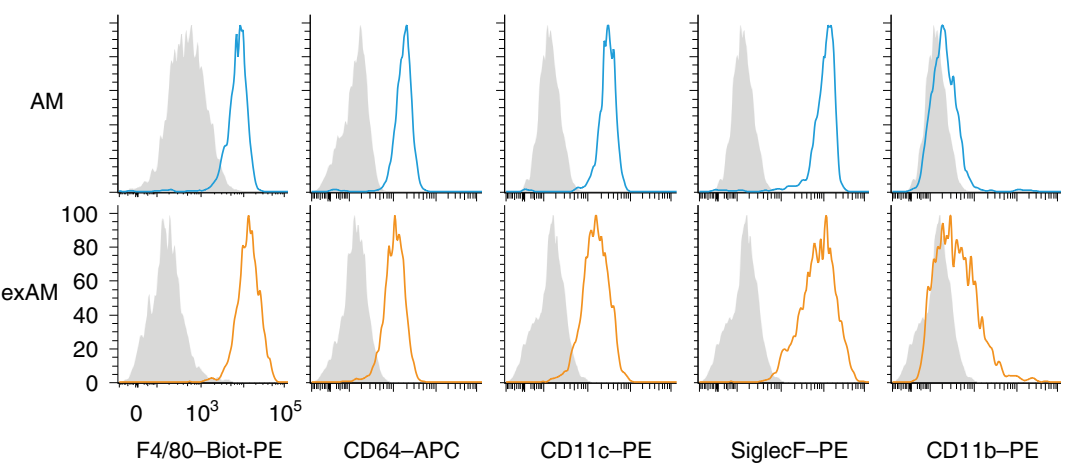

h
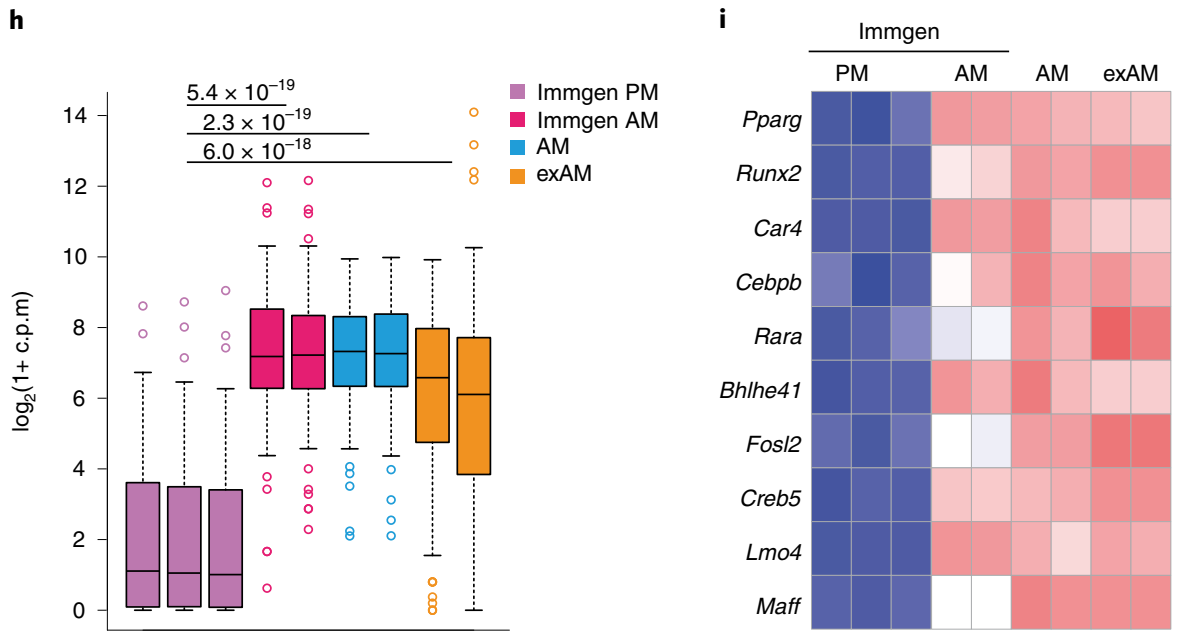


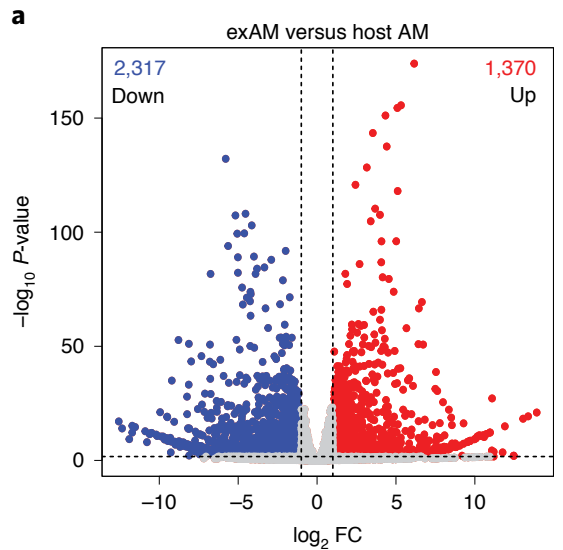

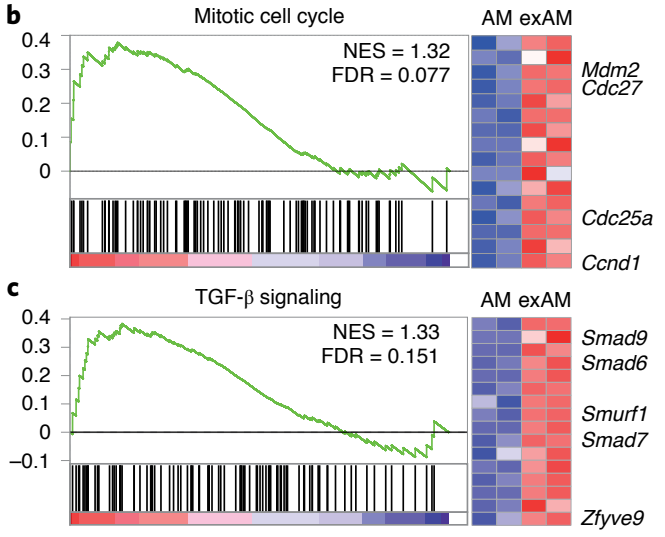

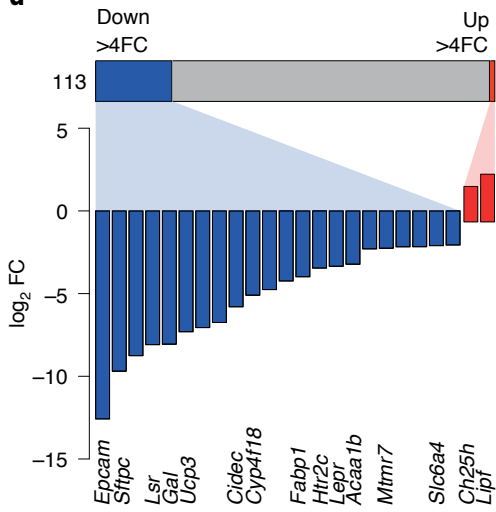

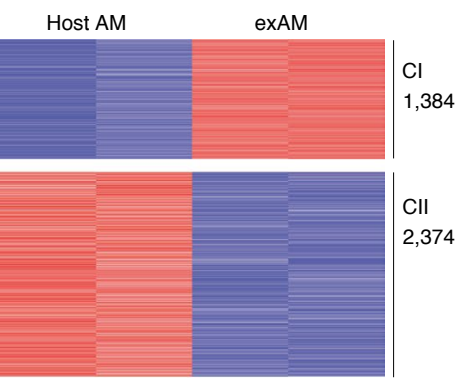

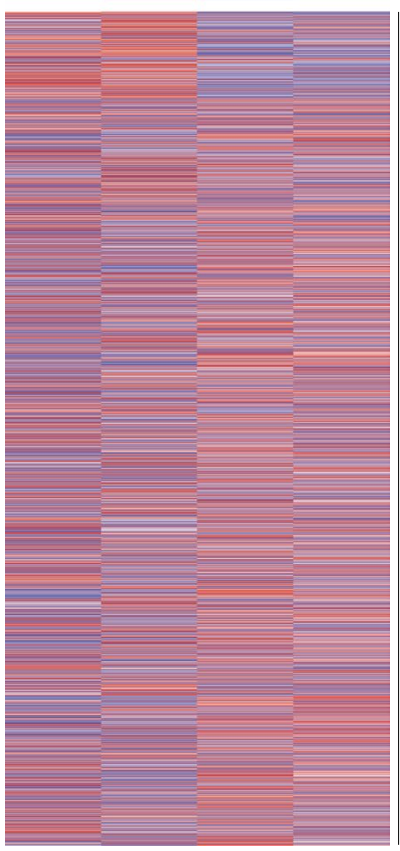

f

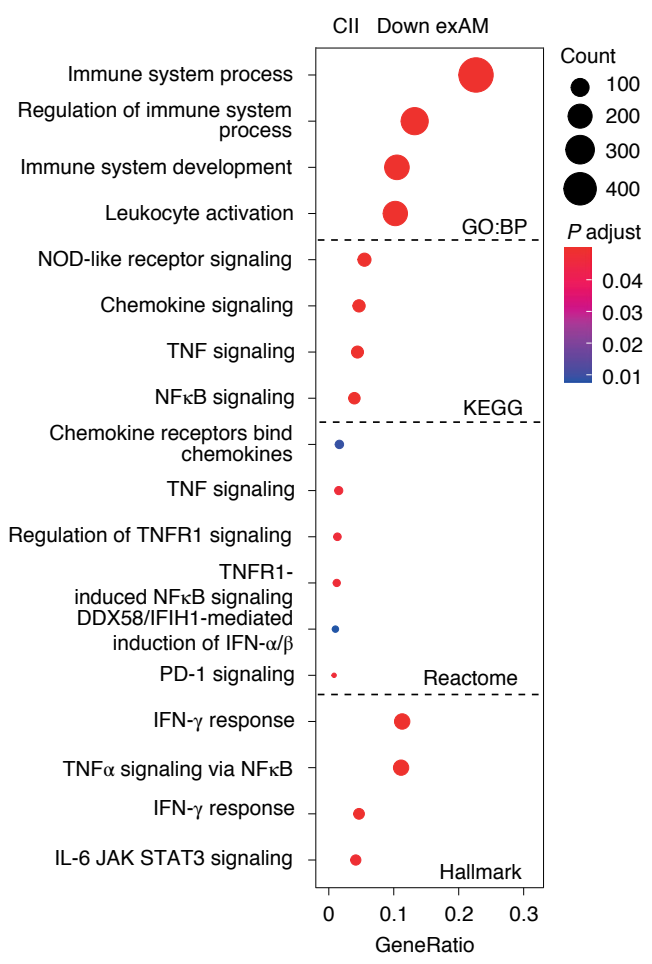

CII Down exAM

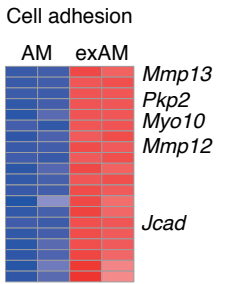

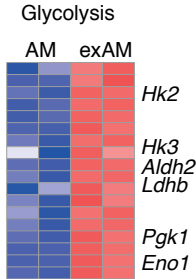

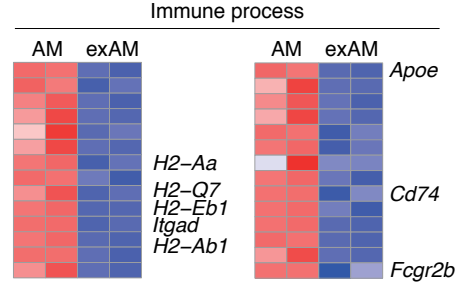

Fig. 3 | Substantial culture adaptations of exAM transcriptome. a, Volcano plot showing differentially expressed genes (DEGs; FC $>2, F D R<0.05$ ) between exAMs (2 months) and in vivo AMs (host AMs). b, Gene set enrichment analysis (GSEA) enrichment plot for GO biological process (GO:BP) positive regulation of mitotic cell cycle gene set (GO:0045931) in AMs versus exAMs. Corresponding leading-edge gene expression heat map shown for AMs versus exAMs. NES, normalized enrichment score. c, GSEA for Reactome:Signaling by TGF- $\beta$ family members gene set (R-HAS-9006936) in AMs versus exAMs. Corresponding leading-edge gene expression heat map shown for AMs versus exAMs. d, DEGs of AMs or exAMs in core AM signature genes ${ }^{3}(F C>4, F D R<0.05)$. e, $K$-means clustering $(K=3)$ heat map showing total genes in AM versus exAM. $\mathbf{f}$, GO analysis on $k$-means clusters $\mathrm{Cl}$ and $\mathrm{CIl}$ using GO:BP, KEGG, Reactome and Hallmark databases. TNF, tumor necrosis factor IL, interleukin. $\mathbf{g}$, Heat maps of gene expression of selected representative key genes on major $\mathrm{GO}$ terms from analysis shown in $\mathbf{f}$. All heat maps showing rlog normalized values from minimum (blue) to maximum (red).

between texAMs and host AMs were not due to the retention of culture-specific gene expression in vivo, but rather to experimental noise or to transplantation-specific effects.
Finally, analysis of core AM signature genes ${ }^{3}$ that distinguish AMs from other macrophages showed that expression of the few genes related to lipid metabolism, surfactant and adhesion mole- 
cules (such as Lsr, Sftpc and Epcam) that were lost in exAMs compared to host AMs, was fully restored in texAMs, with nearly no detectable differences to the other in vivo AM samples (Fig. 4h). Similarly, expression of genes with uniquely low expression levels in AMs that were upregulated in exAMs compared to in vivo AM samples was also restored to low levels in texAMs (Fig. 4h). Together, this showed that the transcriptomic adaptations of long-term cultured exAMs to the culture environment were reversible and could be restored to the characteristic signature of resident AMs upon exposure to the cues of the natural niche environment in vivo.

Epigenetic changes of exAMs in culture are restored in vivo. We next investigated the epigenetic mechanisms underlying the full restoration of transcriptomic AM identity and loss of the transcriptional culture adaptations of texAMs and used ATAC-seq analysis to identify potential changes in chromatin accessibility. When comparing open chromatin regions (OCRs) of cultured exAMs to freshly isolated AMs (BAL AMs) from pools of three biological replicates, we observed a high correlation between replicates, but substantial differences between conditions (Extended Data Fig. 6). After bioinformatic pooling of replicates, we detected 723 lost and 1,504 gained OCRs in exAMs compared to BAL AMs (Fig. 5a), highlighting substantial differences between the in vivo and culture conditions and excluding the possibility of unchanged chromatin accessibility during culture adaptation.

Gene Ontology (GO) term analysis of the genes associated with these OCR alterations showed that terms for metabolism, cellular proliferation, cell adhesion and migration were enriched, whereas immune response terms were depleted in exAMs compared to BAL AMs (Fig. 5b), underscoring similar terms of adaptation to the cell culture environment as the transcriptomic analysis. As individual examples, peaks not present in AM were induced in exAMs in the regulatory regions of $C c n d 1$ (encoding cyclin D1) and $M d m 2$, two cell-cycle genes; $S p h k 1$ and $P k p 2$, two genes important for cell adhesion; and $H k 1$ and $H k 2$, two critical regulators of glycolysis (Fig. 5c). In addition, OCRs of several genes important in lipid metabolism (Acaalb and Cidec) and the immune response, such as several major histocompatibility (MHC) class II genes ( $\mathrm{H} 2-\mathrm{Aa}, \mathrm{H} 2-\mathrm{Ab} 1$ and H2-Eb1), were not detected in exAMs compared to BAL AMs (Fig. $5 c)$.

LPS-stimulated hematopoietic stem cells (HSCs) show transcriptional changes that quickly come back to normal, but conserve a long-term epigenetic memory of the stimulus ${ }^{28}$. This demonstrates that environmental cues can induce long-lasting epigenetic changes, despite the full reversibility of the transcriptional alterations induced by these cues ${ }^{28}$. To test whether the culture-induced epigenetic changes in exAMs were conserved upon transfer into the lung microenvironment in vivo, we transplanted CD45.2 exAMs cultured for 2 months into CD45.1 mice and compared texAMs and host AMs 4 months after transplantation. Whereas the comparison of exAMs to host AMs showed 1,492 gained and 937 lost OCRs, similar to the exAM to BAL AM comparison, we observed nearly identical OCRs in texAMs compared to host AMs (Fig. 5a), indicating that culture-specific alterations in chromatin accessibility were nearly fully restored upon transplantation of exAMs into the lung alveolar niche. Among the 2,500 differential OCRs detected between exAMs and host AMs, only 15 were conserved in texAMs compared to host AMs (Fig. 5d). Consistently, about 1,000 differential OCRs were detected between exAMs and texAMs, whereas BAL AMs and host AMs showed a nearly identical OCR profile (Extended Data Fig. 7). A heat map of unsupervised cluster analysis also indicated the similarity between all in vivo samples, including texAMs, and a strong difference between the in vivo samples and exAMs in culture (Extended Data Fig. 8). Notably, analysis of individual OCRs showed that the peaks in the regulatory regions of cell cycle, adhesion or glycolysis genes in exAMs were not detected in texAMs, whereas the peaks in immune response genes that were not detected in exAMs were re-established in texAMs (Fig. 5c).

Finally, analysis of regulatory regions for AM-specific core transcription factors and surface markers identified AM-specific OCRs that were not detected in other myeloid cells, such as red pulp macrophages (RPM) and microglia or other immune cells (Fig. 5e), identifying them as markers of epigenetic AM identity. For example, AM-specific peaks of the transcription factor Pparg, a key regulator of $\mathrm{AM}$ identity ${ }^{22}$, were not detected in other macrophage populations or immune cells, whereas the surface marker Itgax (CD11c), which is expressed in AMs and other cell types, showed AM-specific and general OCRs (Fig. 5e). Notably, these key regulatory regions of AMs identity did not change across samples and were equally present in BAL AMs and host AMs, exAMs and texAMs (Fig. 5e), indicating that epigenetic AM identity was not lost in culture. In addition, analysis of the transcription factor binding motifs associated with the differential OCRs between exAMs and host AMs revealed that binding sites for the core myeloid PU.1, C/ EBP and RUNX transcription factors were associated with both the differential gained and lost OCRs in exAMs (Extended Data Fig. 9), suggesting that the changes in chromatin accessibility might occur on pre-existing myeloid enhancer platforms. Together, the analysis of epigenetic chromatin accessibility indicated that AM identity was conserved through expansion in culture and that adaptations to the culture environment were transient, reversible and restored to the in vivo status upon transplantation into the alveolar niche.

exAMs show long-term reconstitution of empty AM niche in vivo. To test whether exAMs could reconstitute an empty AM niche, we used Csf $2 \mathrm{rb}^{-1-}$ mice, which lack the $\beta$ chain of the GM-CSF receptor, are deficient in AMs and can be reconstituted with monocytes or macrophages from different sources ${ }^{7,8,26,29,30}$. We transferred $0.4 \times 10^{5}$ cells of 4-month CD45.1 exAM cultures into neonatal Csf2rb ${ }^{-/-}$ mice by intranasal transplantation. After 8 months nearly all donor cells in the lung of $C s f 2 r^{-1-}$ mice had a SiglecF ${ }^{+} \mathrm{CD} 11 \mathrm{c}^{+} \mathrm{AM}$ phenotype and yielded about 0.6 million cells in the BAL, similar to CD45.1 wild-type mice of the same age, whereas untransplanted Csf2rb ${ }^{-/-}$mice had no SiglecF ${ }^{+} \mathrm{CD} 11 \mathrm{c}^{+}$AM (Fig. 6a,b). BAL cells

Fig. 4 | exAMs restore full transcriptional AM identity in vivo. a, Flow cytometric analysis showing contribution of CD45.2 tAMs or texAMs and CD45.1 host AMs to the SiglecF+ ${ }^{+} \mathrm{CD}_{11 \mathrm{c}^{+}}$AM population. Each symbol denotes one mouse $(n=6)$. $\mathbf{b}$, Flow cytometric analysis showing expression of surface markers CD64, CD11c, SiglecF, CD11b and MHCII in host AMs, tAMs and texAMs. Data are representative of three independent experiments. c, Heat map of Spearman's correlation matrix of exAM, host AM, tAM and texAM RNA-seq samples, each with two replicates from pools of three mice each (inter-sample correlation Extended Data Fig. 3).d, Three-dimensional PCA showing tAMs (purple), texAMs (red) and host AMs (blue) compared to various tissue macrophage subsets ${ }^{4}$. KC, Kupffer cell; RPM, red pulp macrophage; PM, peritoneal macrophage; SI M, small intestinal macrophage; LI $M$, large intestinal macrophage. e, Overlaid volcano plot showing DEGs (FC $>2$ and FDR $<0.05)$ in tAMs versus texAMs compared to exAMs versus texAMs (top) and texAMs versus host AMs compared to exAMs versus host AMs (bottom). f, Venn diagram showing overlap of DEGs for exAMs or tAMs compared to texAMs. $\mathbf{g}$, Venn diagram showing total DEGs on pairwise comparisons on all in vivo AMs. $\mathbf{h}$, Heat map showing core AMs up and down signature gene expression ${ }^{3}$ from our data compared to published tissue macrophage subsets ${ }^{4}$ showing rlog normalized values from minimum (blue) to maximum (red). Highlighted boxes indicate restoration of in vivo gene expression of several core AM upregulated genes shown to be altered in culture. 
from exAM-transplanted mice and CD45.1 wild-type mice had the same AM morphology by brightfield microscopy and staining with Diff-Quik dye of cytospin samples (Fig. 6c). A similar full reconstitution of the AM niche by exAMs was also observed 3 months after transplantation in Csf2rb ${ }^{-1-}$ mice (Fig. $6 \mathrm{~b}$ and Extended Data Fig. 10), This demonstrated a strong expansion and self-renewal potential of exAMs in vivo to repopulate an empty alveolar niche and maintain homeostatic numbers long-term.
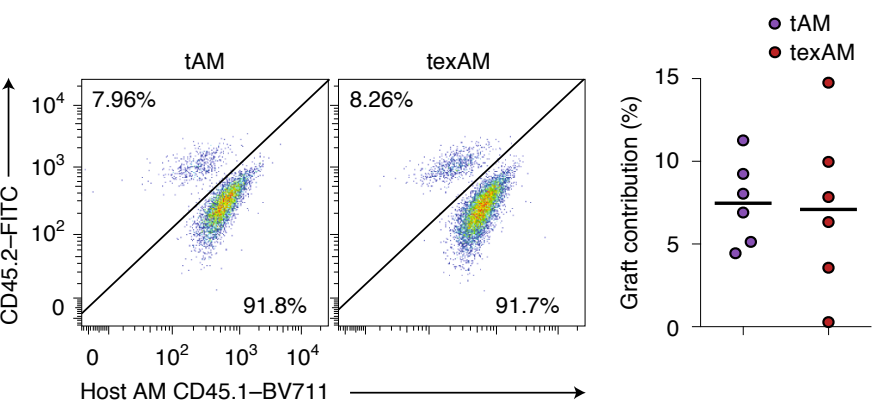

c
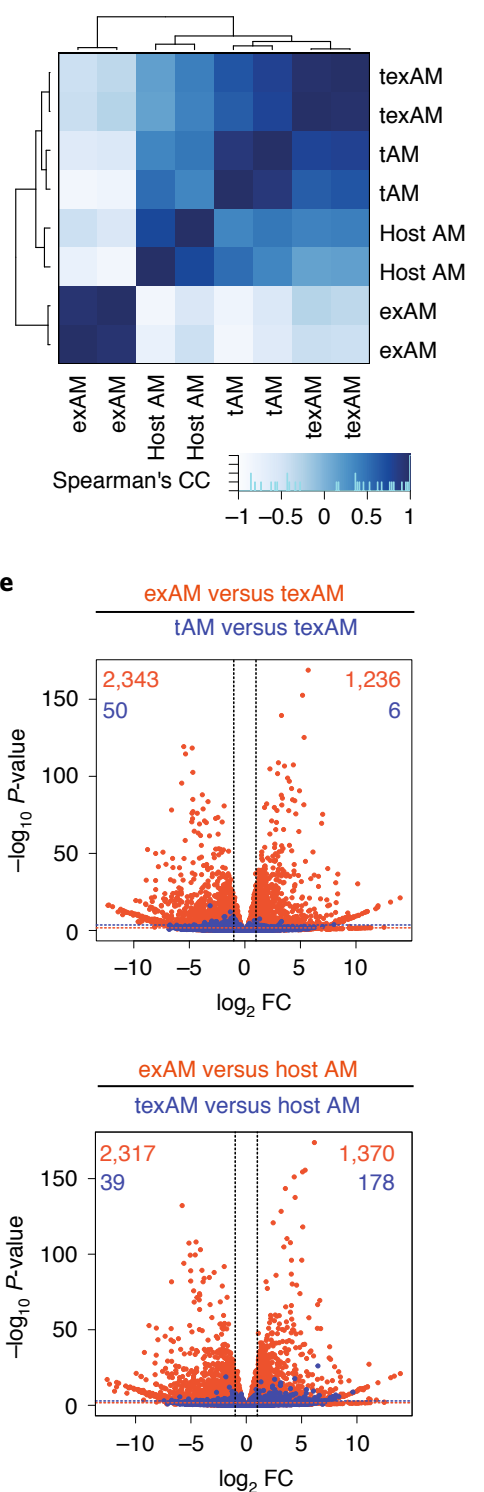

d

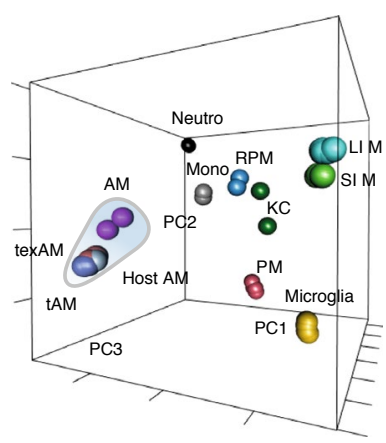

$\mathbf{f}$

exAM versus texAM

tAM versus texAM

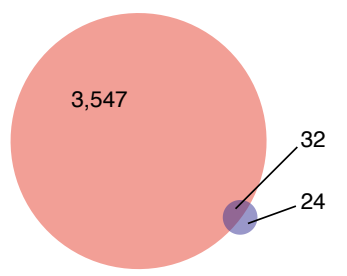

g

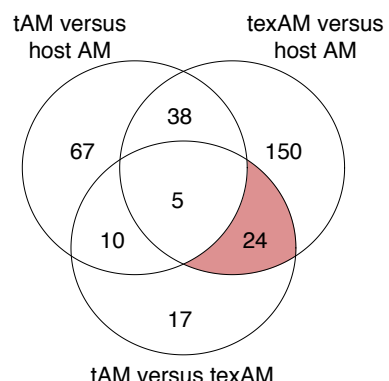

Owing to the complete absence of AMs, Csf2 $r b^{-/-}$mice develop pulmonary alveolar proteinosis (PAP) that is characterized by accumulation of mucus and debris in the alveolar lung fluid ${ }^{29,30}$. Microscopic inspection (Fig. 6c) or flow cytometric analysis (Fig. $6 \mathrm{~b}, \mathrm{~d})$ showed a strong reduction in debris in the lungs of the $C s f 2 r b^{-1-}$ mice that received exAMs compared to untreated Csf2rbmice (Fig. 6c,d), which in some cases resulted in complete clearance, similar to wild-type control mice (Fig. 6c,d). Thus, exAMs b

- Host AM

- tAM

- texAM

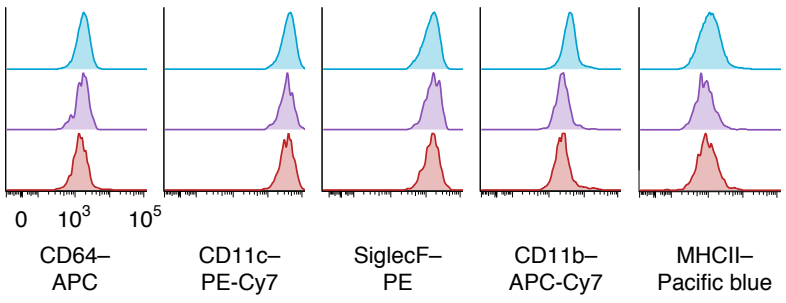

h

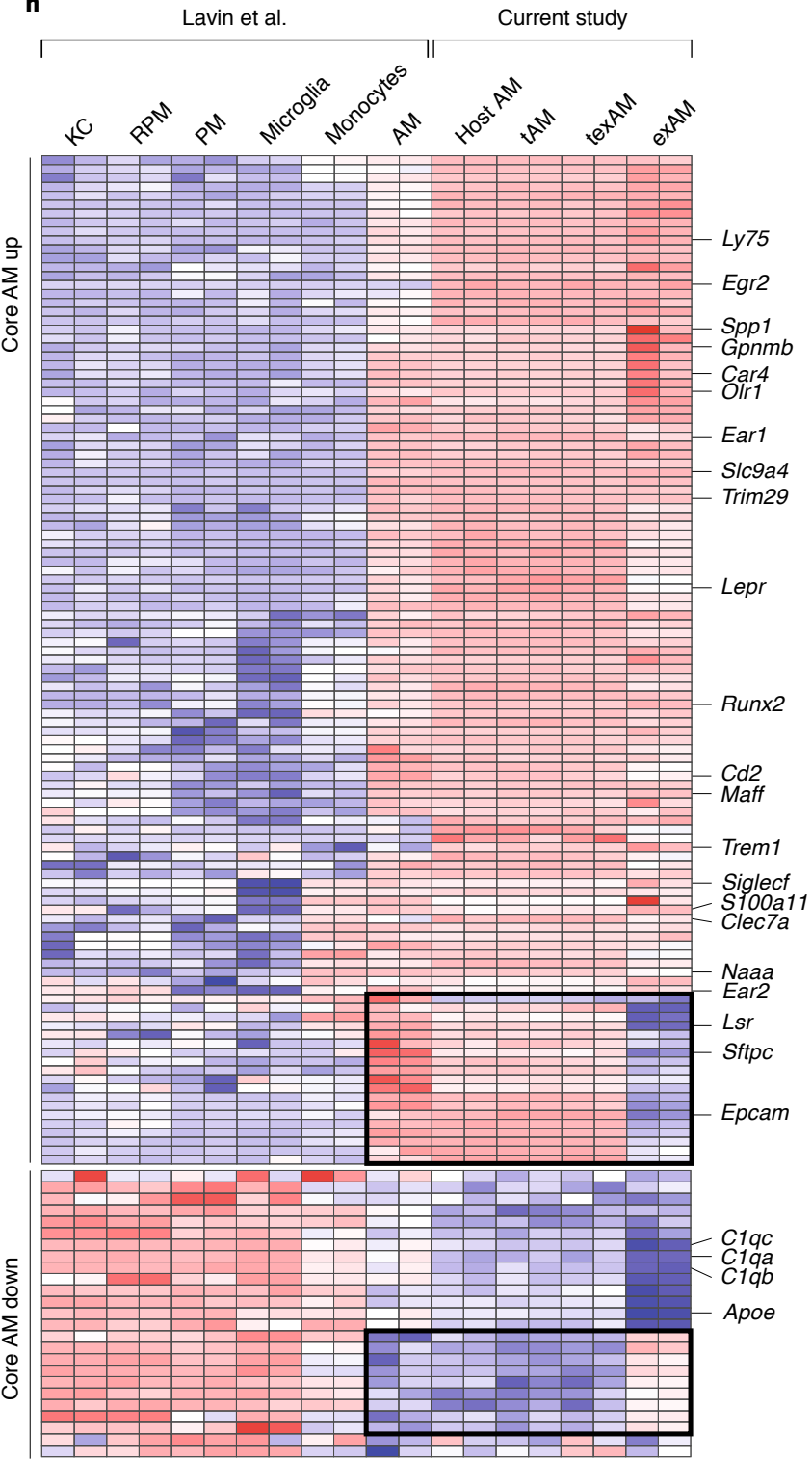



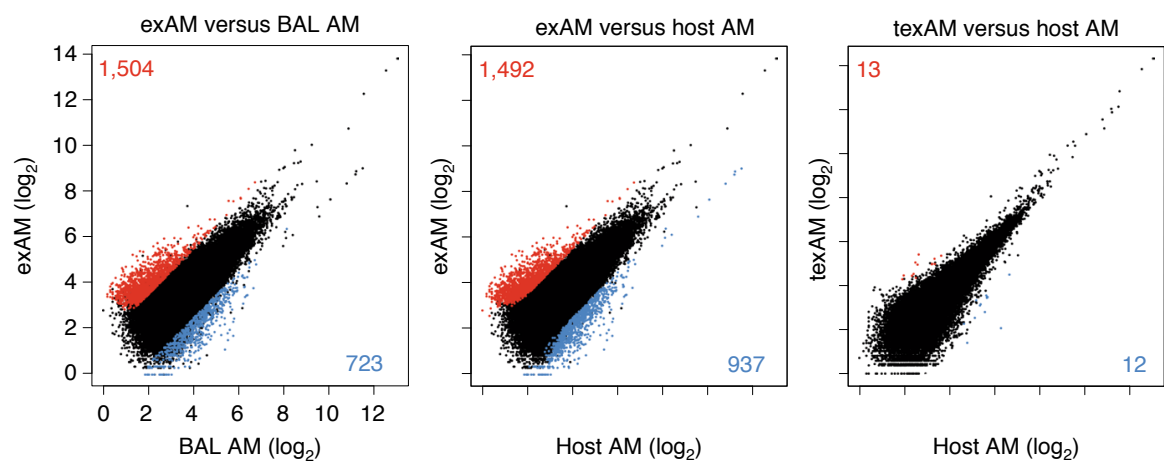

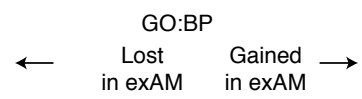

Metabolic process !

Response to stress

Regulation mitotic cell cycle

Actin filament organization

Regulation cell shape!

Regulation cell growth

Regulation cell migration

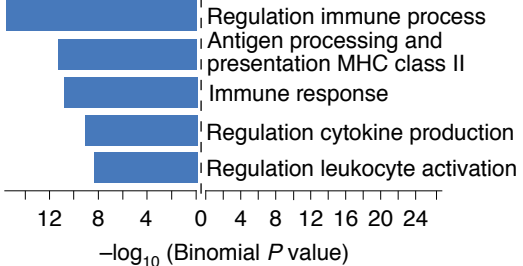

c
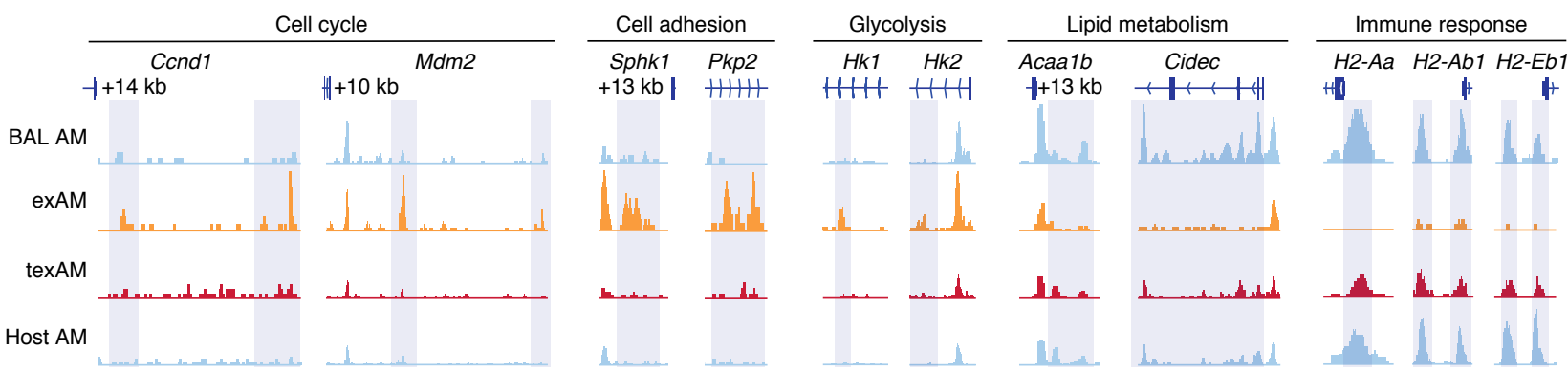

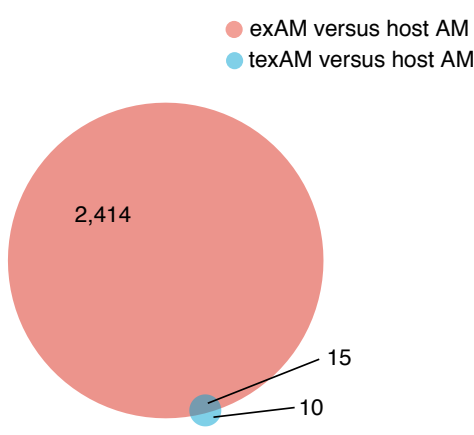

e

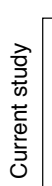

ฏ)

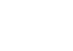

monocytes
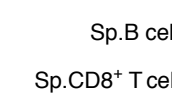

Sp.CD4 ${ }^{+}$T cells

BM.CD11 ${ }^{+}$NK cells

BM.LT-HSC

HprtGapdh

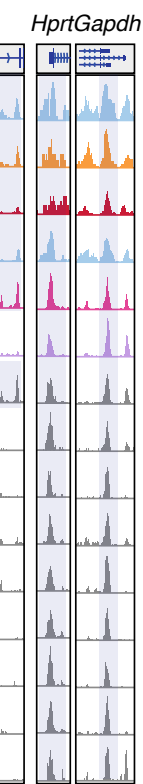

Fig. 5 | Epigenetic changes of exAMs in culture are restored in vivo. a, Scatter-plot analysis of differentially gained (red) or lost (blue) ATAC-seq peaks $\left(\log _{2} \mathrm{FC}>1.5, \mathrm{FDR}<0.05\right)$ in exAMs (2 months) or texAMs 4 months after transplantation compared to BAL AMs or host AMs. Total peaks $(48,100)$ were pooled from three replicates of pools from three mice/cultures each. Each point represents the normalized average signal for each comparison in $\log _{2} . \mathbf{b}, \mathrm{GO}$ analysis using GREAT tool ${ }^{49}$ on differential OCRs in exAMs versus host AMs. c, Examples of integrative genomics viewer (IGV) tracks for key genes linked to GO terms shown in Fig. 5b and genes from Figs. 3d,g.d, Venn diagram showing total differential peaks for exAMs or texAMs compared to host AMs. e, Examples of IGV tracks showing OCRs for AM-specific transcription factors, cell-surface markers and housekeeping genes in our samples alongside various cell populations available from the Immgen consortium ${ }^{50}$. ATAC-seq signal pile-up traces were generated by pooling three replicates from pools of three mice/cultures each. Sp, spleen; BI, blood; BM, bone marrow; RPM, red pulp macrophage; pDC, plasmacytoid dendritic cell; NK, natural killer; LT-HSC, long-term hematopoietic stem cell.

can repopulate an empty alveolar niche in vivo, self-maintain homeostatic numbers in the long-term and perform homeostatic functions.

\section{Discussion}

Here we have shown that mouse AMs could be maintained through long-term culture and could be expanded massively without losing 
a
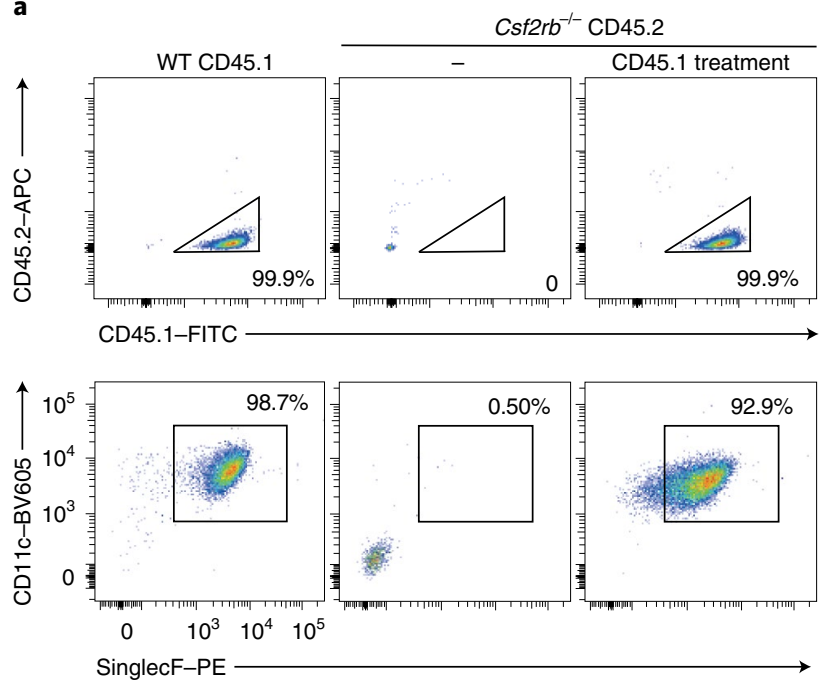

c

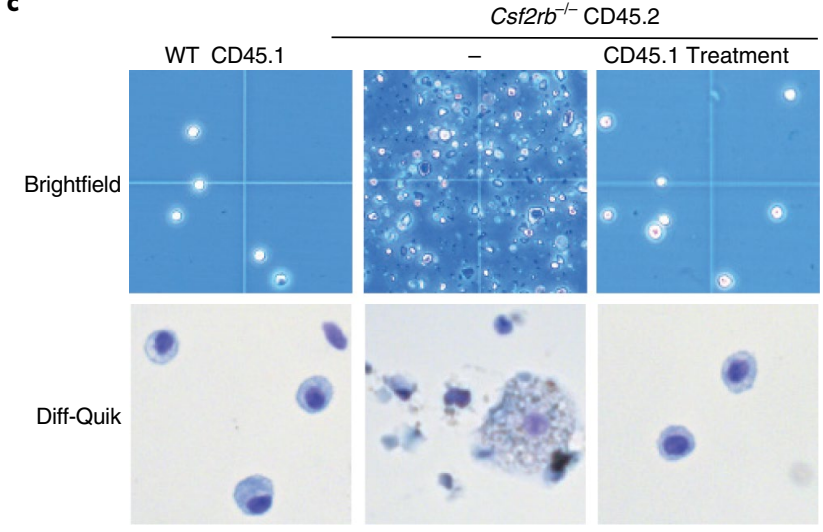

b
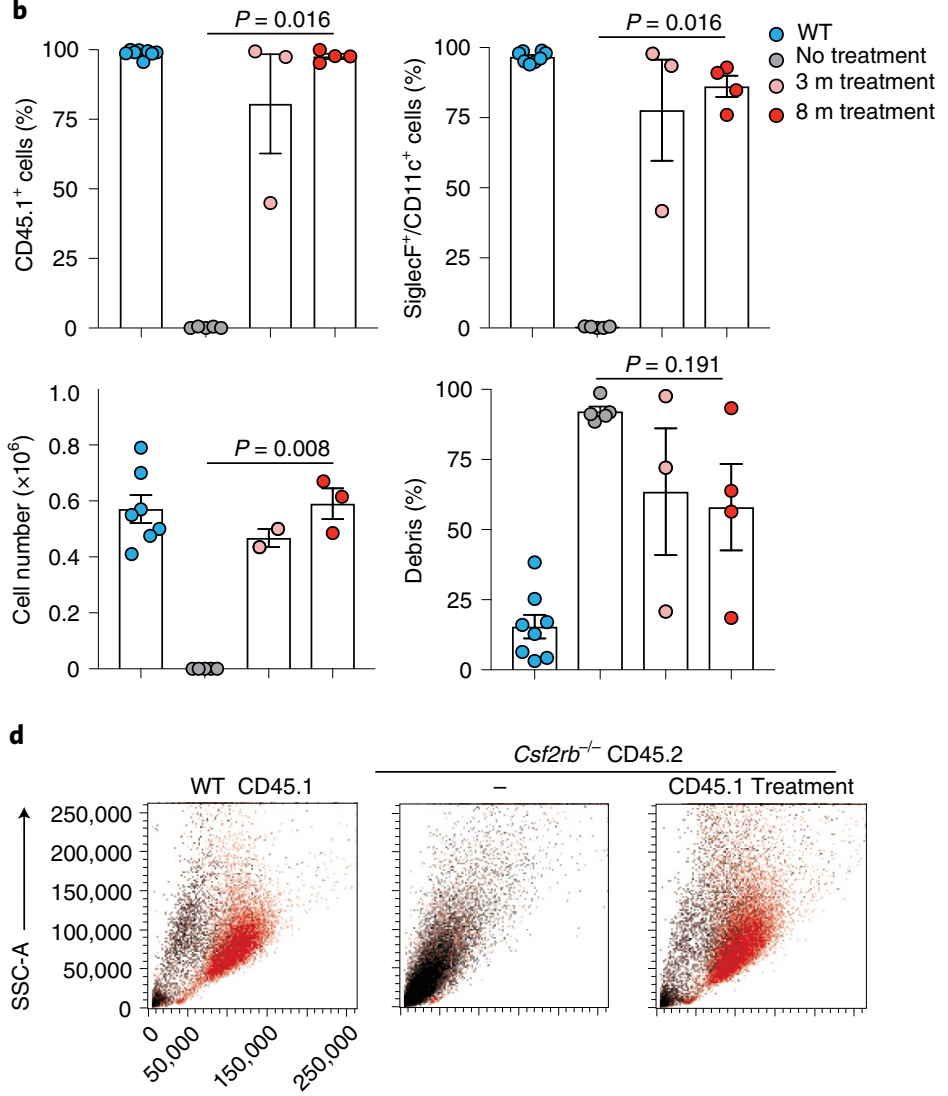

Ssf2rb $^{-/-}$CD45.2
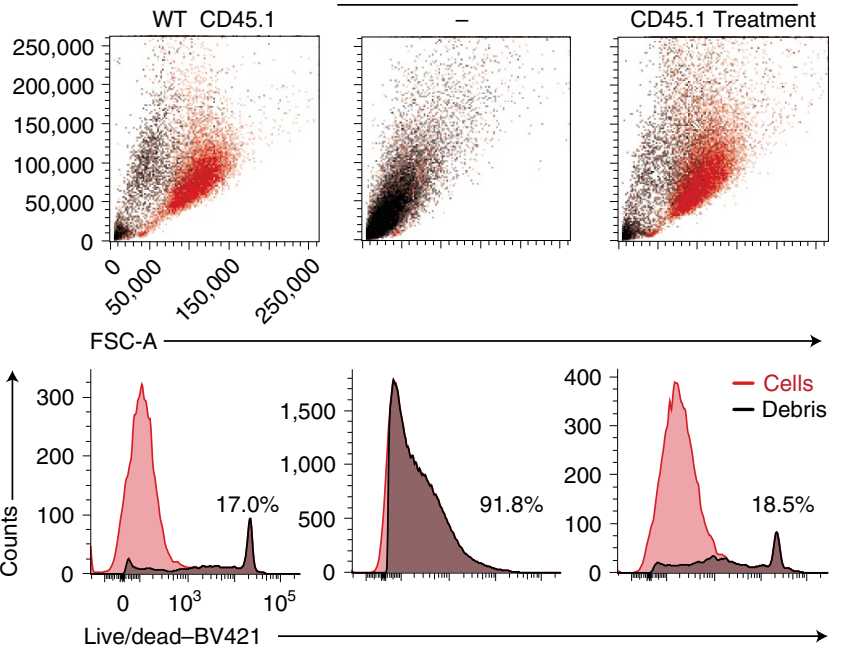

Fig. 6 | exAMs show long-term reconstitution of empty AM niche in vivo. a, Flow cytometric analysis of exAM contribution to Csf2rb ${ }^{-/-}$lungs at 8 months after exAM transplantation. FACS plots showing CD45.1 contribution of engrafted texAM (top) and SiglecF and CD11c AM surface marker expression (bottom). b, Quantification of flow cytometric analysis of CD45.1+, SiglecF+CD11c ${ }^{+} \mathrm{AMs}$, debris/dead cells in BAL fluid or absolute cell counts in BAL fluid. c, Brightfield and Diff-Quik-stained images of BAL at 8 months after transplantation. d, Flow cytometric analysis of debris in BAL of Csf2rb-/- mice 8 months post exAM transplantation. Scatter-plot (top) and histogram (bottom) FACS plots showing debris clearance in exAM-transplanted Csf2rb-/- mice, quantified in $\mathbf{b}$. Data from a,c,d and Extended Data Fig. 10 are representative of two experiments at 3 or 8 months after exAM transplantation quantified in b with each symbol denoting one mouse, $n=7$ (WT), 7 (NT), 2 or 3 (3-month treatment) and 4 (8-month treatment), shown as mean \pm s.e.m. $P$ values are derived by two-tailed unpaired Mann-Whitney U-test.

tissue-resident macrophage identity. Although substantial epigenetic and transcriptional culture adaptations occurred, they were fully reversible upon transplantation into the natural alveolar niche in vivo, indicating a robust cell-endogenous epigenetic setup that was stable through many cell divisions and provided the flexibility to adapt to different environmental cues without losing identity.

It has been suggested that cultured macrophages do not reflect macrophage identity in vivo ${ }^{11}$, because they undergo major changes in gene expression, cytokine responsiveness and metabolism in culture ${ }^{9,10,12}$. Here we confirmed adaptation of AMs to the culture environment with substantial changes in cell adhesion, migration, proliferation, metabolism and immune response-related gene expression, signaling pathways and chromatin accessibility. These changes, however, were fully reversible and restored to the in vivo condition upon transplantation into the alveolar niche. This confirmed a potent role of the microenviron- ment ${ }^{2}$ but raised the question of how AMs conserve the ability to respond to in vivo environmental cues after long periods in culture. Differential OCRs between culture and in vivo environments were enriched for binding sites for the core myeloid PU.1, C/EBP and RUNX transcription factors, which establish a core enhancer platform to determine macrophage identity ${ }^{31}$ and on which additional signal-induced transcription factors can converge ${ }^{32,33}$. This suggests that a stable enhancer architecture, on which accessory transcription factors can activate environment-specific gene expression, maintains cellular identity through culture. Consistent with this, key OCRs and expression of genes for AM-specific transcription factors like Car4 and Pparg, or surface markers like CD11c and SiglecF were maintained throughout culture. This indicates that a highly stable AM-specific identity is maintained throughout culture, while allowing a spectrum of responsiveness to environmental cues and adapted gene expression in vivo and in 
culture, in line with the high degree of plasticity of macrophages in responding to diverse stimuli ${ }^{34}$.

Our observations extend previous reports on the ability of mature macrophages to self-renew while in a differentiated state ${ }^{18,19,35}$. Here we show that AMs can be expanded extensively in culture for extended periods and still assume full epigenetic identity and functionality upon re-integration in their in vivo environment. This suggests that neither culture adaptations, nor long-term proliferation, erase the epigenetic and transcriptional identity of AMs. Such behavior is characteristic of stem cells, such as pluripotent or epithelial stem cells, which can be expanded extensively or indefinitely in culture and still functionally integrate into a tissue environment in vivo ${ }^{13,36}$.

The observed full restoration of normal chromatin accessibility in vivo is distinctly different from scenarios of trained immunity. Although both resident ${ }^{37}$ and monocyte-derived ${ }^{21,27} \mathrm{AMs}$ can undergo trained immunity, the underlying mechanism might be different from culture adaptation. In HSCs, LPS stimulation induces short-term transcriptional changes but long-term epigenetic memory through a TRIF- and C/EBPb-dependent signaling axis ${ }^{28}$. In monocytes, $\beta$-glucan stimulation can induce trained immunity through epigenetic changes in an Akt, mTOR and a glycolysis-dependent manner ${ }^{38-40}$. The PI3K-Akt, mTOR and C/ $\mathrm{EBPb}$ pathways, as well as glycolysis, were induced in exAMs, but changes in chromatin accessibility were completely restored after re-transplantation in vivo, indicating that these pathways might be necessary but not sufficient for the induction of epigenetic memory. FOS and JUN (AP-1) transcription factors have been linked to the process of converting transient environmental signals into long-term epigenetic memory ${ }^{41}$. We did not detect FOS and JUN binding sites by motif enrichment analysis of differential OCRs, which might explain why OCR changes acquired in culture were not maintained after re-transplantation in vivo.

In practical terms, exAMs provide a nearly unlimited source of genetically unmodified and untransformed normal macrophages. As a reliable long-term cell culture system, which does not depend on complex differentiation protocols and could start cultures after freeze-thaw, it has the convenience of a cell line without its shortcomings. In addition, the possibility to shuttle between culture and in vivo environments without loss of cellular identity provides a unique experimental system for screening, biochemical and genetic investigation with direct in vivo validation potential.

Finally, our results have highly promising implications for potential macrophage cellular therapies. Whereas $\mathrm{T}$ cell therapy has been largely limited to hematological cancers ${ }^{16}$, macrophages as major constituent of the tumor stroma have a high potential to treat solid tumors. Furthermore, macrophages fitted with a chimeric antigen receptor have shown promise in animal models ${ }^{42,43}$. The use of macrophage cellular therapy has so far been limited by the difficulty to grow large numbers of macrophages in culture ${ }^{17}$. Our results demonstrate that large-scale and long-term expansion of macrophages ex vivo is possible, without compromising the identity of transplanted macrophages in vivo, a feature that is critical both for efficacy and safety considerations. Beyond cancer, the robustness of macrophage identity through culture might be applied to cellular therapies in infectious disease and regenerative medicine ${ }^{44}$, including degenerative liver disease ${ }^{45}$ and $\mathrm{PAP}^{30,46}$. Furthermore, it might be useful in the study of respiratory diseases, including COVID-19, where macrophages play important roles in disease development ${ }^{47}$.

\section{Online content}

Any methods, additional references, Nature Research reporting summaries, source data, extended data, supplementary information, acknowledgements, peer review information; details of author contributions and competing interests; and statements of data and code availability are available at https://doi.org/10.1038/ s41590-022-01146-w.
Received: 27 July 2021; Accepted: 20 January 2022; Published online: 24 February 2022

\section{References}

1. Davies, L. C., Jenkins, S. J., Allen, J. E. \& Taylor, P. R. Tissue-resident macrophages. Nat. Immunol. 14, 986-995 (2013).

2. Bleriot, C., Chakarov, S. \& Ginhoux, F. Determinants of resident tissue macrophage identity and function. Immunity 52, 957-970 (2020).

3. Gautier, E. L. et al. Gene-expression profiles and transcriptional regulatory pathways that underlie the identity and diversity of mouse tissue macrophages. Nat. Immunol. 13, 1118-1128 (2012).

4. Lavin, Y. et al. Tissue-resident macrophage enhancer landscapes are shaped by the local microenvironment. Cell 159, 1312-1326 (2014).

5. Guilliams, M. \& Svedberg, F. R. Does tissue imprinting restrict macrophage plasticity? Nat. Immunol. 22, 118-127 (2021).

6. Sieweke, M. H. Waddington's valleys and Captain Cook's islands. Cell Stem Cell 16, 7-8 (2015).

7. van de Laar, L. et al. Yolk sac macrophages, fetal liver, and adult monocytes can colonize an empty niche and develop into functional tissue-resident macrophages. Immunity https://doi.org/10.1016/j.immuni.2016.02.017 (2016).

8. McQuattie-Pimentel, A. C. et al. The lung microenvironment shapes a dysfunctional response of alveolar macrophages in aging. J. Clin. Invest. https://doi.org/10.1172/JCI140299 (2021).

9. Gosselin, D. et al. Environment drives selection and function of enhancers controlling tissue-specific macrophage identities. Cell 159, 1327-1340 (2014).

10. Gosselin, D. et al. An environment-dependent transcriptional network specifies human microglia identity. Science https://doi.org/10.1126/science. aal3222 (2017).

11. Svedberg, F. R. et al. The lung environment controls alveolar macrophage metabolism and responsiveness in type 2 inflammation. Nat. Immunol. 20, 571-580 (2019).

12. Bohlen, C. J. et al. Diverse requirements for microglial survival, specification, and function revealed by defined-medium cultures. Neuron 94, 759-773 (2017)

13. Fuchs, E. The impact of cell culture on stem cell research. Cell Stem Cell 10, 640-641 (2012)

14. Helft, J. et al. GM-CSF mouse bone marrow cultures comprise a heterogeneous population of $\mathrm{CD} 11 \mathrm{c}\left({ }^{+}\right) \mathrm{MHCII}\left({ }^{+}\right)$macrophages and dendritic cells. Immunity 42, 1197-1211 (2015).

15. Misharin, A. V., Saber, R. \& Perlman, H. Eosinophil contamination of thioglycolate-elicited peritoneal macrophage cultures skews the functional readouts of in vitro assays. J. Leukoc. Biol. 92, 325-331 (2012).

16. Lim, W. A. \& June, C. H. The principles of engineering immune. Cells Treat. Cancer Cell 168, 724-740 (2017).

17. Dolgin, E. Cancer-eating immune cells kitted out with CARs. Nat. Biotechnol. 38, 509-511 (2020).

18. Aziz, A., Soucie, E., Sarrazin, S. \& Sieweke, M. H. MafB/c-Maf deficiency enables self-renewal of differentiated functional macrophages. Science 326, 867-871 (2009)

19. Soucie, E. L. et al. Lineage-specific enhancers activate self-renewal genes in macrophages and embryonic stem cells. Science 351, aad5510 (2016).

20. Busch, C. J., Favret, J., Geirsdottir, L., Molawi, K. \& Sieweke, M. H. Isolation and long-term cultivation of mouse alveolar macrophages. Bio. Protoc. https:// doi.org/10.21769/BioProtoc.3302 (2019).

21. Aegerter, H. et al. Influenza-induced monocyte-derived alveolar macrophages confer prolonged antibacterial protection. Nat. Immunol. 21, 145-157 (2020).

22. Schneider, C. et al. Induction of the nuclear receptor PPAR- $\gamma$ by the cytokine GM-CSF is critical for the differentiation of fetal monocytes into alveolar macrophages. Nat. Immunol. 15, 1026-1037 (2014).

23. Rauschmeier, R. et al. Bhlhe 40 and Bhlhe41 transcription factors regulate alveolar macrophage self-renewal and identity. EMBO J. 38, e101233 (2019).

24. Angelidis, I. et al. An atlas of the aging lung mapped by single cell transcriptomics and deep tissue proteomics. Nat. Commun. 10, 963 (2019).

25 . Yu, X. et al. The cytokine TGF- $\beta$ promotes the development and homeostasis of alveolar macrophages. Immunity 47, 903-912 (2017).

26. Misharin, A. V. et al. Monocyte-derived alveolar macrophages drive lung fibrosis and persist in the lung over the life span. J. Exp. Med. 214, 2387-2404 (2017)

27. Machiels, B. et al. A gammaherpesvirus provides protection against allergic asthma by inducing the replacement of resident alveolar macrophages with regulatory monocytes. Nat. Immunol. 18, 1310-1320 (2017).

28. de Laval, B. et al. C/EBP $\beta$-dependent epigenetic memory induces trained immunity in hematopoietic stem cells. Cell Stem Cell 26, 793 (2020).

29. Happle, C. et al. Pulmonary transplantation of macrophage progenitors as effective and long-lasting therapy for hereditary pulmonary alveolar proteinosis. Sci. Transl. Med. 6, 250ra113 (2014).

30. Suzuki, T. et al. Pulmonary macrophage transplantation therapy. Nature https://doi.org/10.1038/nature13807 (2014). 
31. Heinz, S. et al. Simple combinations of lineage-determining transcription factors prime cis-regulatory elements required for macrophage and B cell identities. Mol. Cell 38, 576-589 (2010).

32. Heinz, S., Romanoski, C. E., Benner, C. \& Glass, C. K. The selection and function of cell type-specific enhancers. Nat. Rev. Mol. Cell Biol. 16, 144-154 (2015).

33. Glass, C. K. \& Natoli, G. Molecular control of activation and priming in macrophages. Nat. Immunol. 17, 26-33 (2016).

34. Xue, J. et al. Transcriptome-based network analysis reveals a spectrum model of human macrophage activation. Immunity 40, 274-288 (2014).

35. Imperatore, F. et al. SIRT1 regulates macrophage self-renewal. EMBO J. 36, 2353-2372 (2017).

36. Green, H. Cultured cells for the treatment of disease. Sci. Am. 265, 96-102 (1991).

37. Yao, Y. et al. Induction of autonomous memory alveolar macrophages requires $\mathrm{T}$ cell help and is critical to trained immunity. Cell 175, 1634-1650 (2018).

38. Bekkering, S. et al. Metabolic induction of trained immunity through the mevalonate pathway. Cell 172, 135-146 (2018).

39. Saeed, S. et al. Epigenetic programming of monocyte-to-macrophage differentiation and trained innate immunity. Science 345, 1251086 (2014).

40. Cheng, S. C. et al. mTOR- and HIF- $1 \alpha$-mediated aerobic glycolysis as metabolic basis for trained immunity. Science 345, 1250684 (2014).

41. Larsen, S. B. et al. Establishment, maintenance, and recall of inflammatory memory. Cell Stem Cell 28, 1758-1774 (2021).
42. Klichinsky, M. et al. Human chimeric antigen receptor macrophages for cancer immunotherapy. Nat. Biotechnol. 38, 947-953 (2020).

43. Morrissey, M. A. et al. Chimeric antigen receptors that trigger phagocytosis. eLife https://doi.org/10.7554/eLife.36688 (2018).

44. Chan, M. W. Y. \& Viswanathan, S. Recent progress on developing exogenous monocyte/macrophage-based therapies for inflammatory and degenerative diseases. Cytotherapy 21, 393-415 (2019).

45. Starkey Lewis, P. J., Moroni, F. \& Forbes, S. J. Macrophages as a cell-based therapy for liver disease. Semin. Liver Dis. 39, 442-451 (2019).

46. Happle, C. et al. Pulmonary transplantation of human induced pluripotent stem cell-derived macrophages ameliorates pulmonary alveolar proteinosis. Am. J. respiratory Crit. Care Med. 198, 350-360 (2018).

47. Merad, M. \& Martin, J. C. Pathological inflammation in patients with COVID-19: a key role for monocytes and macrophages. Nat. Rev. Immunol. 20, 355-362 (2020).

48. Gal-Oz, S. T. et al. ImmGen report: sexual dimorphism in the immune system transcriptome. Nat. Commun. 10, 4295 (2019).

49. McLean, C. Y. et al. GREAT improves functional interpretation of cis-regulatory regions. Nat. Biotechnol. 28, 495-501 (2010).

50. Yoshida, H. et al. The cis-regulatory atlas of the mouse immune system. Cell 176, 897-912 (2019).

Publisher's note Springer Nature remains neutral with regard to jurisdictional claims in published maps and institutional affiliations.

(C) The Author(s), under exclusive licence to Springer Nature America, Inc. 2022 


\section{Methods}

Animals. C57BL/6 CD $45.1^{+}$and CD $45.2^{+}$mice were obtained from Charles River and Janvier, respectively. Csf2 $\mathrm{rb}^{-/-}$mice were kindly provided by $\mathrm{M}$. Greter (University of Zurich) and described previously $y^{51}$. Mice of 2-4 months of age were used in all experiments. In transplantation experiments, the age of donor and recipient mice was between 6 and 8 weeks. Mice were housed under specific-pathogen-free conditions maintaining temperatures of $21{ }^{\circ} \mathrm{C}$ with $50 \%$ humidity in individually ventilated cages in a controlled 12 -h reverse light/ dark cycle and were provided with food and water ad libitum. Only female mice were used for transplantation in both RNA-seq and ATAC-seq experiments. For neonatal transplants, animals of both sexes were used. In vivo procedures were performed following the protocols approved by the ethics committee of no. 014, Marseille, France (APAFIS 3292-2015122109359224), the TU Dresden and the Landesdirektion Sachsen in accordance with institutional, national and European animal welfare legislation (TVV28/2018 AKZ, IC 114-G0160/16 and DD24.1-5131/449/30)

Isolation and ex vivo expansion of alveolar macrophages. Isolation of AMs from BAL was performed as previously described ${ }^{20}$. The expansion and maintenance of exAMs ex vivo was performed as described previously ${ }^{20}$. AMs were isolated from both male and female mice. Briefly, cells were plated at a density of $1.1-1.5 \times 10^{6}$ per $10-\mathrm{cm}$ Petri dish in $10 \mathrm{ml}$ complete medium. The medium was supplemented with murine $2-5 \%$ GM-CSF supernatant from J558L cells transfected with murine GM-CSF. Cultures of exAMs were passaged every $3-4 \mathrm{~d}$ and cultured for 2-4 months for functional assays or transplantation studies.

Preparation of lung homogenates for flow cytometric analysis. Mice were killed by cervical dislocation, lungs were collected, cut into small pieces and incubated with $1 \mathrm{mg} \mathrm{ml}^{-1}$ collagenase-2 (CLS2, Worthington) and $0.15 \mathrm{mg} \mathrm{ml}^{-1}$ DNaseI (DN25, Sigma) at $37^{\circ} \mathrm{C}$ for $30 \mathrm{~min}$ with constant agitation. Cell suspension was filtered through a 70- $\mu \mathrm{m}$ mesh and erythrocytes were removed by RBC lysis (RBC lysis buffer, cat. no. 00-4333-57 Invitrogen).

Flow cytometry. Lung cell suspensions was pre-incubated with $\mathrm{Fc}$ receptor blocking antibody (clone 2.4 G2, BD Pharmingen or TruStain FcX ${ }^{\mathrm{TM}}$, BioLegend) and Zombie fixable cell viability dye (NIR cat. no. 77814, Aqua cat. no. 77143 or UV cat. no. 77474; BioLegend) or DAPI (D9542, Sigma) for $15-20 \mathrm{~min}$ at $4{ }^{\circ} \mathrm{C}$. For antibody staining, cells were incubated with antibody cocktail for $20 \mathrm{~min}$ at $4^{\circ} \mathrm{C}$. FACS sorting and analysis was conducted with FACS Canto, LSRII and LSR Fortessa systems (BD). The following antibodies were used for staining cells: anti-CD11b (clone M1/70, eBioscience cat. no. 12-0112-82 1:500 dilution or BD cat. no. 557657 1:400 dilution), anti-CD11c (clone N418, eBioscience cat. no. 120114-82 1:500 dilution or BioLegend cat. no. 117334 1:200 dilution; B-Ly6, BD cat. no. 563929 1:300 dilution; HL3, BD cat. no. 550261 1:200 dilution), anti-F4/80 (clone BM8, eBioscience cat. no. 13-4801-82 1:200 dilution or BioLegend cat. no. 123141 1:200), anti-MHCII (clone M5/114.15.2, eBioscience cat. no. 48-5321-80 1:400 dilution), anti-CD45.1 (clone A20, BD cat. no. 563982 1:200 dilution or BioLegend cat. no. 110730 1:750 dilution, cat. no. 110722 1:200 dilution, cat. no. 110706 1:200 dilution), anti-CD45.2 (clone 104, eBioscience or BioLegend cat. no. 139308 1:200 dilution, cat. no. 109814 1:200 dilution), anti-Ly6C (clone AL21, BD cat. no. 553104 1:400 dilution), anti-CD64 (clone X54-5/7.1, BioLegend cat. no. 139304 1:200 dilution or BD cat. no. 558539 1:200 dilution), anti-SiglecF (clone E50-2440, BD cat. no. 552126 1:200 dilution), anti-B220 (clone RA3-6B2, eBioscience cat. no. 12-0452-82 1:200 dilution, cat. no. 13-0452-82 1:200, cat. no. 17-0452-83 1:200 dilution), Fc receptor blocking antibody (clone 2.4 G2, BD Pharmingen cat. no. 553142 1:200 dilution or TruStain FcX ${ }^{\mathrm{TM}}$, BioLegend cat. no. 101320 1:200 dilution). Diva software was used for acquisition of data and FlowJo software (v.10 Treestar) for data analysis. GraphPad Prism software (v.9.0) was used for statistical analysis.

Intratracheal and intranasal transplantation of alveolar macrophages. Mice were anesthetized by isoflurane inhalation, placed on their back, the tongue was gently pulled out and $0.8-1 \times 10^{6}$ fresh AMs or exAMs in $80-100 \mu \mathrm{l}$ PBS were instilled intratracheally using $1-\mathrm{ml}$ syringe with a blunt $22-\mathrm{G}$ gavage needle. Mice were observed while recovering from anesthesia and then returned to their cages for routine care and handling. Neonatal Csf2 $\mathrm{rb}^{-1-}$ mice (postnatal day 3) were anesthetized with isoflurane and transplanted with $0.4 \times 10^{5} \mathrm{AM}$ resuspended in PBS and in a total volume of $7 \mu \mathrm{l}$.

Ex vivo alveolar macrophage assays. Cytospins and in-well photography. AMs were plated at a density of approx. $0.4 \times 10^{5} \mathrm{cells} \mathrm{cm}^{-2}$ for $4 \mathrm{~h}$ for attachment in either six-well or 100-mm nontreated cell culture dishes. Brightfield images were acquired from well center using Zeiss Axio Vert A1 microscope at $\times 10$ magnification. Fresh BAL AMs or exAMs (2 months culture, detached and counted) were subjected to cytocentrifugation at 300-450 r.p.m. for 4 min using a Cytospin 4 (Thermo Fisher) and subsequent Diff-Quik staining following the manufacturer's protocols (cat. no. 9990700, Thermo Fisher Scientific Rapid-chrome Kwik-diff kit). Images were acquired using the three-dimensional Histech slide scanner and images were processed using Qupath (v.0.2.0) with built-in ImageJ software. For Csf2rb ${ }^{-/-}$ transplants, brightfield images were taken with an inverted microscope (Dmil, Leica) after transfer of BAL cells to a Neubauer counting chamber. Cell suspensions were cytospun at 800 r.p.m. for $3 \mathrm{~min}$ using a Cytospin 4 (Thermo Fisher), followed by Kwik-diff staining according to the manufacturer's instructions (cat. no. 9990700, Thermo Scientific Rapid-chrome Kwik-diff kit). Images were taken with a Zeiss Axiolab A1 microscope.

Apoptosis assay. exAMs were plated at a density of $1 \times 10^{5}$ cells per well of a nontreated 24-well plate (Nunc). As positive control for apoptotic cells, apoptosis was induced in exAM using $1 \mu \mathrm{M}$ staurosporine (S5921, Sigma) for $1 \mathrm{~h}$ at $37^{\circ} \mathrm{C}$ and $5 \% \mathrm{CO}_{2}$. Untreated exAMs or apoptotic exAMs were stained using the PE annexin V apoptosis detection kit I (cat. no. 559763, BD Pharmingen) according to manufacturer's protocols and analyzed by flow cytometry. Percentage of early (annexin $\mathrm{V}^{+} / 7-\mathrm{AAD}^{-}$) and late apoptotic (annexin $\mathrm{V}^{+} / 7-\mathrm{AAD}^{+}$) cells was determined on total cells.

Bacterial killing assay. AMs were plated overnight at a density of $1 \times 10^{6}$ cells per well of a nontreated 96-well plate (Nunc). K Pneumoniae wild-type strain Kp52.145 was obtained from the Institut Pasteur collection ${ }^{52,53}$. The bacteria were grown overnight in $\mathrm{LB}$ medium at $37^{\circ} \mathrm{C}$ in a Minitron shaker incubator (180 r.p.m., $\mathrm{CH}-$ 4103, Infors AG). The bacteria were precultured until they reached a log phase of growth after which they were incubated with the AMs at multiplicity of infection of 100 for $15 \mathrm{~min}$ at $37^{\circ} \mathrm{C}$ and $5 \% \mathrm{CO}_{2}$. The supernatant was discarded and cell lysates were collected at 0,30 and $90 \mathrm{~min}$ after incubation with bacteria. Gentamicin solution (1:1,000 dilution, G1272, Sigma) was added to the medium for the 30 - and 90 -min time points to prevent extracellular bacterial replication. The cell lysates were plated in serial dilutions in LB agar and the colonies were estimated the following day.

Cell cycle analysis. AMs were plated at density of $0.2 \times 10^{6}$ cells per well of nontreated 12-well dishes (Nunc). The cells were pulsed with $10 \mu \mathrm{M} \mathrm{EdU} \mathrm{(C10634,}$ Thermo Fisher) for $4 \mathrm{~h}$ at $37^{\circ} \mathrm{C}$ to label proliferating cells. Cells were then detached, washed and pre-incubated with Fc receptor blocking antibody and cell viability dye followed by antibody staining as described under the flow cytometry section. Cells were fixed with $4 \%$ PFA for $20 \mathrm{~min}$ at $4{ }^{\circ} \mathrm{C}$ and permeabilized with Click-iT saponin permeabilization and wash buffer for $15 \mathrm{~min}$ at $4^{\circ} \mathrm{C}$. The Click-iT reaction was performed using the Click-iT Plus EdU Alexa Fluor 647 Flow Cytometry kit (C10634, Thermo Fisher) according to the manufacturer's instructions. For DNA staining, cells were resuspended in permeabilization buffer containing $1 \mu \mathrm{g} \mathrm{ml}^{-1}$ DAPI (D9542, Sigma) for $30 \mathrm{~min}$ at room temperature. FACS data acquisition was conducted with a BD LSR Fortessa and analysis was performed using FlowJo software (v.10 Treestar).

Phagocytosis assay using fluorescent latex beads. AMs were incubated in growth medium containing fluorescent yellow-green, carboxylate-modified polystyrene latex beads (L4655, Sigma-Aldrich) at 1:400 dilution for $1 \mathrm{~h}$ at $37^{\circ} \mathrm{C}$. Supernatant medium containing beads was discarded post-centrifugation and the cells were washed three times for $5 \mathrm{~min}$ with PBS. After cytocentrifugation, cells were fixed using $4 \%$ PFA for $10 \mathrm{~min}$ and permeabilized with $0.1 \%$ Triton-X in PBS for $15 \mathrm{~min}$ at room temperature. Cells were incubated in blocking buffer $(5 \%$ donkey serum $+1 \%$ BSA in PBS) for $15 \mathrm{~min}$ at room temperature followed by Actin Red staining (two drops per $\mathrm{ml}$ in PBS). All the steps were carried out with washing steps in between (three times, 5 min each using PBS). The slides were air-dried and mounted with ProLong Gold Antifade mountant (P36931, Thermo Fisher)

Phagocytosis assay by flow cytometry. AMs were plated for $4 \mathrm{~h}$ and replaced with fresh medium containing $2 \mu \mathrm{g} \mathrm{ml} l^{-1}$ pHrhodo Red Zymosan Bioparticles Conjugate (P35364, Thermo Fisher) and incubated at $37^{\circ} \mathrm{C}$ and for controls at $4{ }^{\circ} \mathrm{C}$ on ice for $2 \mathrm{~h}$. Subsequently, AMs were detached and processed for flow cytometric analysis.

Detection of lysosome and lysosomal active cathepsin B. AMs were incubated with Acridine Orange (1:1,000 dilution of stock solution in PBS, 6130, Bio-Rad) for $20 \mathrm{~min}$ at $37^{\circ} \mathrm{C}$ or with Magic Red solution ( $1 \times$ final concentration, ICT937, Bio-Rad) for $50 \mathrm{~min}$ at $37^{\circ} \mathrm{C}$. Nuclei were stained using Hoechst 33342 solution (1:200 dilution, cat. no. 62249 , Thermo Fisher) for $10 \mathrm{~min}$ at $37^{\circ} \mathrm{C}$. After staining, AMs were mounted on slides and immediately imaged using Zeiss inverse ApoTome microscope and analyzed using FIJI image analysis software (v.1.52n). MagicRed (MR) and Acridine Orange (AO) quantification were performed on images by dividing the number of MR/AO-positive cells by the number of DAPI-positive cells.

Detection of LPS/IFN- $\gamma$-induced ROS and Nitrite generation. BAL AMs or exAMs were seeded at a density of 100,000 cells per well in 96-well plates and stimulated with $100 \mathrm{ng} \mathrm{ml}^{-1}$ E. coli O55:B5 LPS (L4005, Sigma) and $200 \mathrm{U} \mathrm{ml}^{-1} \mathrm{IFN}-\gamma$ (31505 , Peprotech) for $16 \mathrm{~h}$. Then, supernatant was collected for Nitrite measurement using the Griess Reagent system (G2930, Promega) following the manufacturer's instructions (absorbance, $520 \mathrm{~nm}$ ). For determining ROS production, stimulated cells were incubated for $1 \mathrm{~h}$ with $10 \mu \mathrm{m}$ CM-H2DCFDA (C6827, Thermo Fisher), 
followed by measurement of fluorescence emission at $520 \mathrm{~nm}$ after excitation at $492 \mathrm{~nm}$ using a plate reader (Infinite M Plex, Tecan).

RNA sequencing. For exAMs, total RNA was extracted after 2 months of culture. exAMs were transplanted into 2-month-old CD45.1 mice and analyzed 4 months after transplantation. In vivo AM subsets were sorted (live, singlets,

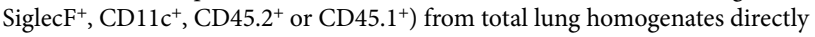
into RNA lysis buffer (RLT buffer, QIAGEN) for RNA extraction using a BD FACSAriaIII machine. RNA-seq samples were generated from a pool of three mice or exAM cultures in duplicates. Amplified complementary DNA was prepared from 2 ng of total RNA using the Ovation RNA-seq system V2 (NuGEN Technologies) following manufacturer's instructions. Briefly, first-strand cDNA was prepared using a combination of random and poly-T DNA/RNA chimeric SPIA (single-primer isothermal amplification) primers and reverse transcriptase. Priming sites were used to synthesize second-strand cDNA using a DNA polymerase, purified using Agencourt RNAClean XP beads (Beckman Coulter) and subjected to SPIA. Amplified cDNA was purified using AMPure XP beads (Beckman Coulter) and $500 \mathrm{ng}$ was fragmented by sonication using a Covaris E210 instrument. Library preparation was performed using Ovation Ultralow Library System kit (NuGEN Technologies) according to manufacturer's instructions. Briefly, $100 \mathrm{ng}$ of amplified cDNA was blunted, phosphorylated and ligated to indexed adaptor dimers. The libraries were then enriched by PCR amplification and surplus PCR primers were removed by purification using AMPure XP beads. DNA libraries were checked for quality using 2100 Bioanalyzer (Agilent) and quantified using Kapa Sybr Fast Light Cycler 480 qPCR kit (Kapa Biosystems) following manufacturer's instructions.

ATAC sequencing. BAL AMs pooled from three mice were expanded ex vivo for 2 months and transplanted intratracheally into lungs of 2-3 WT CD45.1 mice. The lungs were collected at 4 months post transplantation and sorted for CD $45.2^{+}$ exAMs or CD45. $1^{+}$host AMs using a BD FACS AriaIII machine. Then, $5 \times 10^{3}$ cells from three mice each of BAL AMs, exAMs and 2-3 mice each for texAMs or host AMs were pooled and used for ATAC-seq. The ATAC-seq was conducted in technical triplicate transposase reactions, from pools of 2-3 mice for each replicate, again controlling for biological variation. Triplicates were then further pooled bioinformatically for the analyses shown in Fig. 5 (thus representing nine mice each). Fast ATAC-seq protocol was used as described ${ }^{54}$. Cells were lysed with $1 \%$ of digitonin in TD buffer and subjected at the same time to Tn 5 transposase (Nextera). The reactions were incubated for $30 \mathrm{~min}$ at $37^{\circ} \mathrm{C}$. DNA were purified using Cleanup Minelute kit (28204, QIAGEN) according to the manufacturer's protocol. Library were prepared using Nextera DNA Library Preparation kit (FC-121-1030, Illumina) with four cycles of PCR according to the manufacturer's protocol. DNA was quantified with a qPCR using Nextera primer and amplified with a second PCR of 12 to 18 cycles. Libraries were cleaned up with a ratio of 0.7 of AMPure XP beads (Beckman coulter) and quantified with a Qubit fluorometer (Life Technologies). Library quality was assessed by using 2100 Bioanalyzer (Agilent technologies). Libraries were sequenced with 75 paired-ends using NextSeq 500 instrument (Illumina) for an average of $2 \times 10^{7}$ reads per sample

Bioinformatic analysis. RNA-seq analysis. The following analyses were conducted with R (v.3.3). Samples were aligned to mouse genome version mm 10 using Bowtie aligner (v.1.1.2). A table of raw counts was generated using htseq-count (v.0.6.1) and R (v.3.3). All samples were normalized to $1 \mathrm{M}$ reads using the c.p.m. function from the edgeR package. To check reliability of samples replicates, we conducted PCA on normalized expression data on all expressed genes (excluding lowly expressed genes) and correlation tests on normalized expression data. For identification of DEGs, a filtering step was conducted depending on the comparison of interest. Genes having more than 4 c.p.m. in at least one condition in a pairwise comparison were kept for the remaining analysis. For detection of DEGs, raw data were treated using DESeq package (v.1.20). GO analyses were conducted on DEGs after $k$-means clustering between exAMs versus host AMs with the help of the ChIPseeker package (v.1.26). R software (v.3.3) was used to generate heat maps.

ATAC-seq analysis. Data were pooled from three independent experiments. Fastq file quality control was assessed by the fastqc tool. Adaptor trimming was performed using Cutadapt (v.2.6). Bowtie2 (v.2.3.5.1) was used to align and index sequences on the mm10 mouse genome and thus generated the first series of bam files. Then, peak detection was made on these bam files by Genrich (v.0.6.1). Then, from the first series of bam files, a second series was generated as following: after using Bowtie2 (v.2.3.5.1), mitochondrial DNA was removed with Samtools (v.1.9) and Picard (v.2.24.2) was used to remove PCR duplicates. Finally, Samtools allowed to remove non-unique alignments. Bigwig files were generated from BedGraph files using a bdg2bw script (https://gist.github.com/taoliu/2469050). These BedGraph files were created from the second series of bam files. All peaks detected by Genrich from exAM, texAM and host AM BAL samples were pooled and merged together with bedtools (v.2.26.0). Then a table of counts was generated by bedtools from these peaks. The signal was computed from the second series of bam files. Then peaks with $>10$ counts in at least one condition were kept for the following analysis. Opened and closed peaks were detected with the DESeq package (v.1.20). Peaks with adjusted $P$ values $<0.05$ were declared as differentially open between two conditions. Comparisons were texAMs versus host AMs, exAMs versus host AMs and exAMs versus texAMs. Then $\log _{2}(\operatorname{count}+1)$ was represented in scatter-plots comparing conditions two by two. Peaks with $\log _{2} \mathrm{FC}$ computed by DESeq 2 as $>1.5$ (and $<1.5$, respectively) were highlighted in red (respectively in blue).

For motif enrichment analysis, 1,492 peaks were selected as gained in exAMs (as compared to host) and 937 were selected as lost. Then motif analysis was performed on these two groups of peaks using HOMER (v.4.10) (http://homer. salk.edu/homer). HOMER, inter alia, is a discovery motif analyzer and screens for enrichment of known motifs. HOMER perl script findMotifsGenome.pl was used with the mm10 mouse genome as a background (random genomic sequences samples according to GC content of input sequences). Venn diagrams were plotted using VennDiagram package (v.1.6.20). GO analysis and gene associations with peaks were performed with GREAT tool (v.4.0.4) using default options to assign regions and using minimum region-based fold enrichment of 1.2 and term annotation counts $(5-1,000)$ were set as global controls for GO term analysis. For peak visualization reference ATAC-seq sample bigwig files were downloaded from the Immgen consortium ${ }^{50}$ (GSE100738). Peaks were visualized on our samples independently or in parallel with the Immgen references using IGV (v.2.10.2) and were auto-scaled by group.

Reporting Summary. Further information on research design is available in the Nature Research Reporting Summary linked to this article.

\section{Data availability}

All sequencing data generated in this study have been deposited in the Gene Expression Omnibus (GEO) repository under the accession number GSE194144.

\section{References}

51. Robb, L. et al. Hematopoietic and lung abnormalities in mice with a null mutation of the common $\beta$ subunit of the receptors for granulocyte-macrophage colony-stimulating factor and interleukins 3 and 5. Proc. Natl Acad. Sci. USA 92, 9565-9569 (1995).

52. Nassif, X. \& Sansonetti, P. J. Correlation of the virulence of Klebsiella pneumoniae $\mathrm{K} 1$ and $\mathrm{K} 2$ with the presence of a plasmid encoding aerobactin. Infect. Immun. 54, 603-608 (1986).

53. Riottot, M. M., Fournier, J. M. \& Jouin, H. Direct evidence for the involvement of capsular polysaccharide in the immunoprotective activity of Klebsiella pneumoniae ribosomal preparations. Infect. Immun. 31, 71-77 (1981).

54. Corces, M. R. et al. Lineage-specific and single-cell chromatin accessibility charts human hematopoiesis and leukemia evolution. Nat. Genet. 48, 1193-1203 (2016).

\section{Acknowledgements}

We thank L. Chasson for histology (CIML), M. Barad, S. Bigot, A. Zouine (CIML), A. Gompf and K. Bernhardt (CMCB Technology Platform, TU Dresden) for flow cytometry, $\mathrm{M}$. Sohn for sequencing and the K. Rajewsky laboratory for hosting and general support (MDC). We also thank L. Razafindramana, CIML and CRTD Animal facilities for mouse husbandry and the light microscopy facility of the CMCB technology platform, TU Dresden. This study was supported by institutional grants from TU Dresden, the Institut National de la Santé et de la Recherche Médicale, Centre National de la Recherche Scientifique and Aix-Marseille University and grants to M.H.S. from the Agence Nationale pour la Recherche (ANR-17-CE15-0007-01 and ANR-18-CE12-0019-03), Fondation ARC pour la Recherche sur le Cancer (PGA1 RF20170205515), an INSERM-Helmholtz cooperation and the European Research Council under the European Union's Horizon 2020 Research and Innovation program (grant agreement no. 695093 MacAge). S. Subramanian and S.V.A. received doctoral fellowship from the MDC and S.V.A and L.G received doctoral fellowship from the Fondation ARC pour la Recherche sur le Cancer, respectively. K.M. received support from a Human Frontier Science Program long-term fellowship and Stiftung Charité. M.H.S. is an Alexander von Humboldt Professor at TU Dresden (FRA/1188926).

\section{Author contributions}

M.H.S., K.M., S. Subramanian, C.J.B., L.G. and S.V.A. designed experiments and analyzed data. S. Subramanian, C.J.B., K.M. and L.G. performed most experiments. S.V.A., J.F., R.Y.Y.A., M.B. and S.G. contributed to experiments and data analysis. J.M., H.B. and G.G. performed bioinformatic analysis. S. Sarrazin, L.A., B.dL. and P.K.K. supervised experiments and provided advice. S. Subramanian and C.J.B. contributed to writing the manuscript, M.B. and S. Subramanian generated figures. C.J.B. and M.H.S. supervised the project, M.H.S. conceived the project and wrote the manuscript.

\section{Competing interests}

The authors declare no competing interests. 


\section{Additional information}

Extended data is available for this paper at https://doi.org/10.1038/s41590-022-01146-w. Supplementary information The online version contains supplementary material available at https://doi.org/10.1038/s41590-022-01146-w.

Correspondence and requests for materials should be addressed to Michael H. Sieweke.
Peer review information Nature Immunology thanks Andrew Macdonald and the other, anonymous, reviewer(s) for their contribution to the peer review of this work. Ioana Visan was the primary editor on this article and managed its editorial process and peer review in collaboration with the rest of the editorial team.

Reprints and permissions information is available at www.nature.com/reprints. 
a
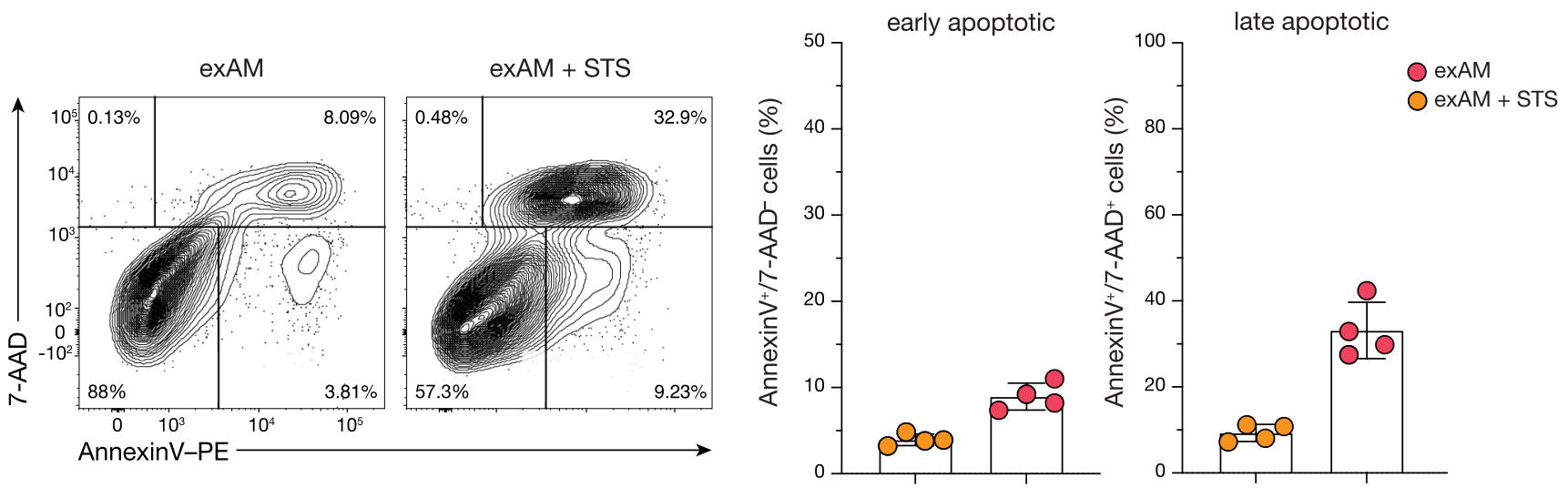

b

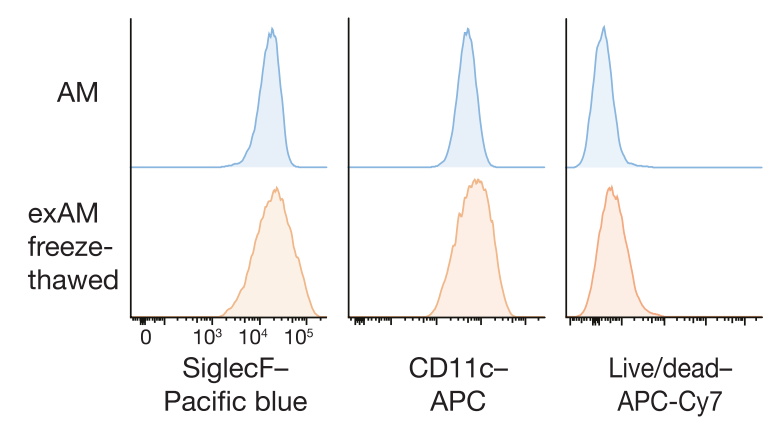

Extended Data Fig. 1 | Freezing exAM does not affect cell death rate or AM phenotype. (a) Flow cytometric analysis of early (annexin $\mathrm{V}^{+} / 7-\mathrm{AAD}^{-}$) and late (annexin $\mathrm{V}^{+} / 7-\mathrm{AAD}^{+}$) apoptotic cell death in 4 months exAM with 1 hour staurosporine (STS) treated exAM as positive control. Data shown as mean \pm s.d. $(n=4)$. (b) Flow cytometric comparison of AM cell-surface markers SiglecF and CD11c and viability dye (Zombie NIR) on freshly isolated AM and freeze-thawed exAM. Frozen exAM were 2 months expanded, 2 years 5 months frozen and expanded 1 month after thawing. Data shown are representative of 2 independent experiments. 
a

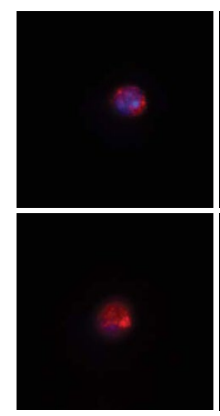

b

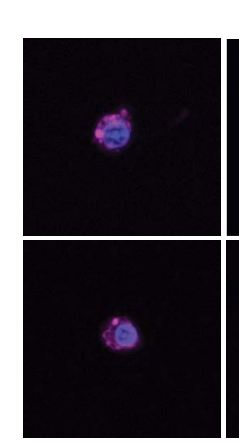

C

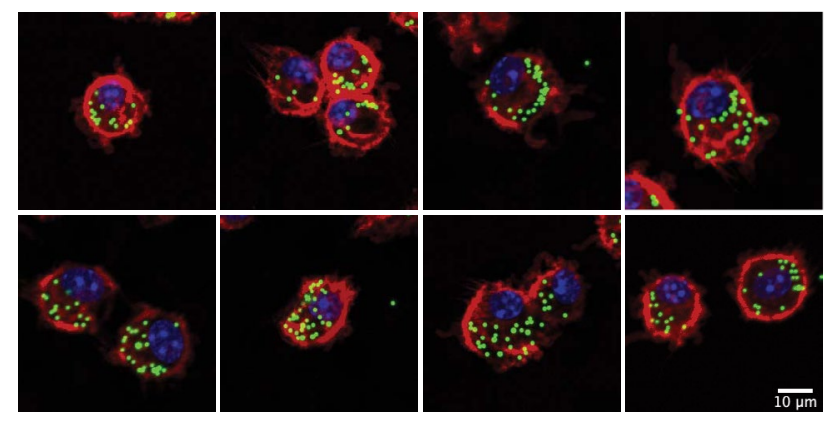

AM

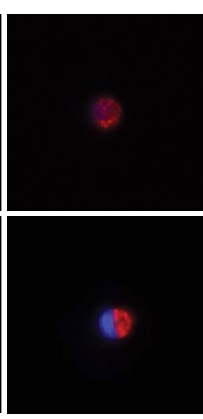

AM

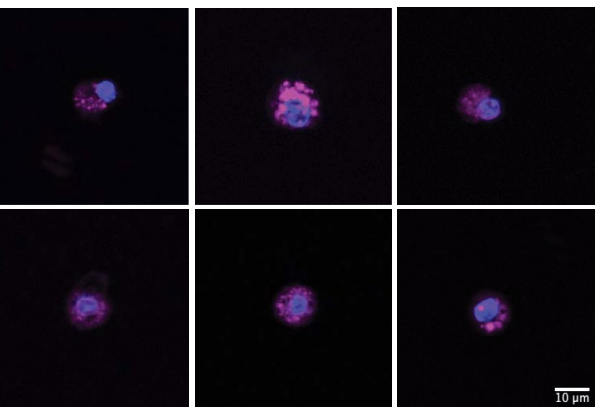

AM

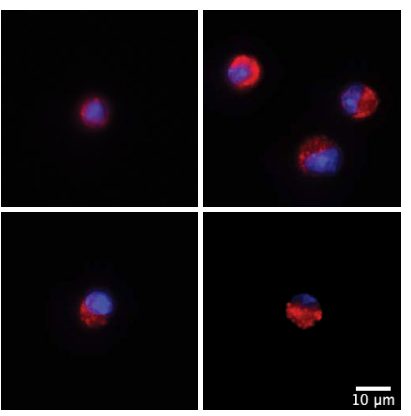

$\overline{10 \mu m}$

$\overline{10 \mathrm{pm}}$

$10 \mu \mathrm{mm}$

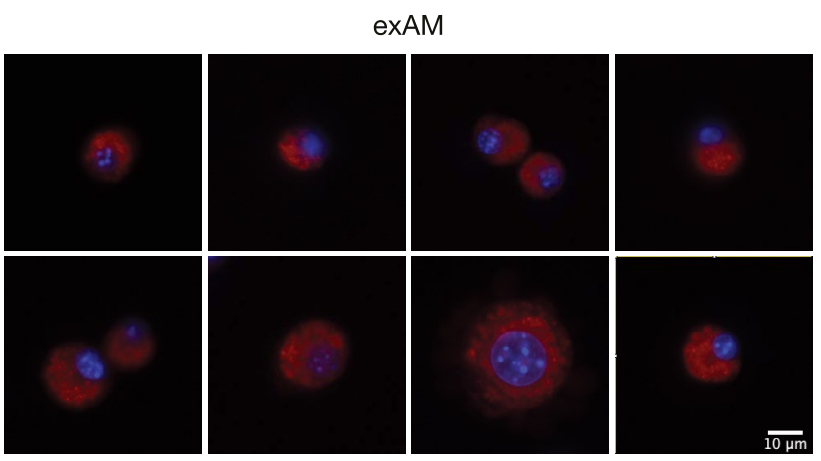

exAM

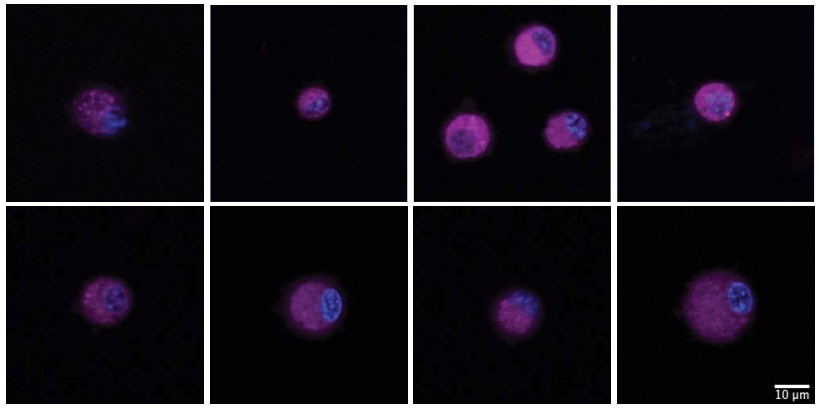

exAM

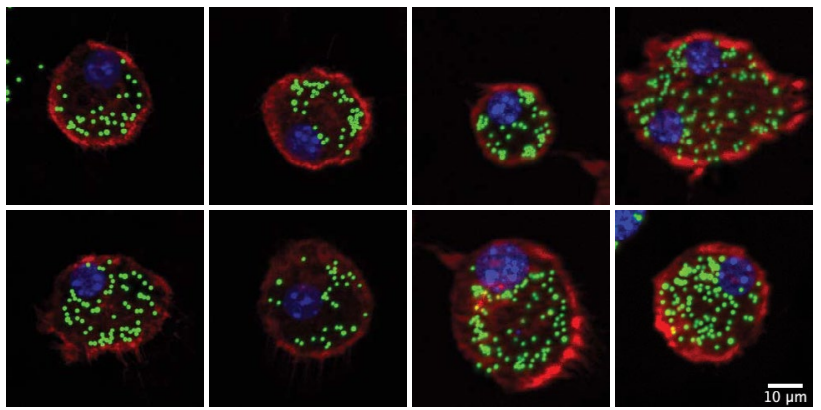

Extended Data Fig. 2 | exAM show lysosome activity and phagocytosis similar to AM. Representative fluorescence images for the lysosomal marker acridine orange (a), lysosomal cathepsin B activity using Magic Red (b) and phagocytosis of green fluorescent latex beads (c) on AM and exAM. Scale bars $10 \mu \mathrm{m}$. Data are representative of 2 independent experiments with at least 3 biological replicates each. 


\section{TECHNICAL REPORT}

NATURE IMMUNOLOGY
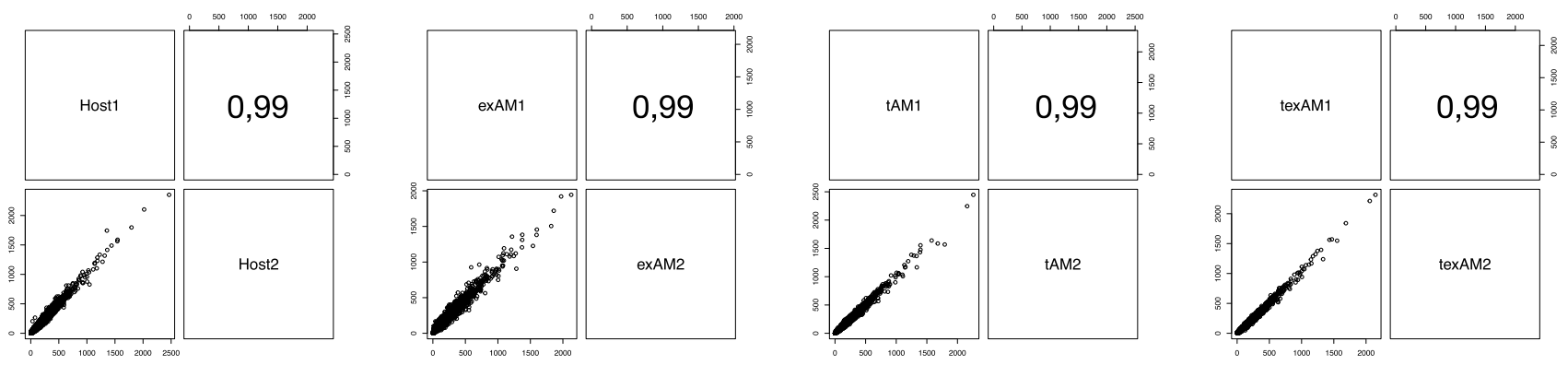

Extended Data Fig. 3 | Pearson correlation of RNA-seq samples. Pearson correlation scatter-plots of replicates from pools of 3 mice each for Host AM, exAM, tAM and texAM RNA-seq samples. 


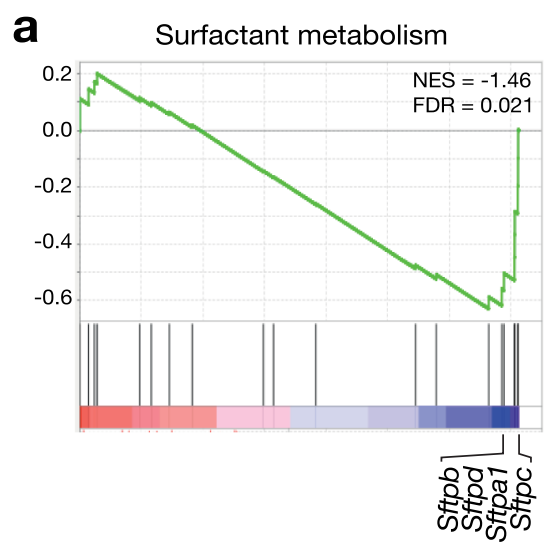

b

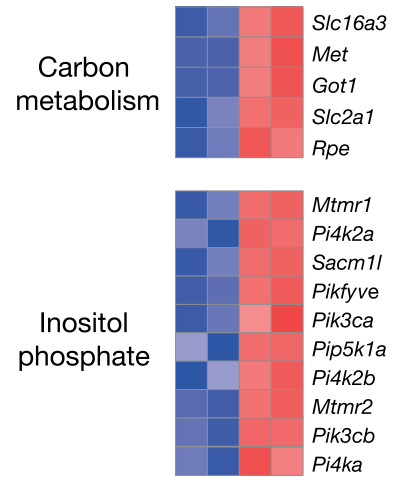

Extended Data Fig. 4 | Transcriptomic adaptation of exAM in response to regulatory cues in the changed microenvironment. (a) Gene set enrichment analysis (GSEA) for Reactome: Surfactant metabolism (R-HAS-9006936) in AM vs exAM. Leading-edge surfactant related genes highlighted below. (b) Gene expression heat maps of key genes from GO terms, "carbon metabolism" and "inositol phosphate signaling" of analysis shown in Fig. 3f, showing rlog normalized values from minimum (blue) to maximum (red). 
a

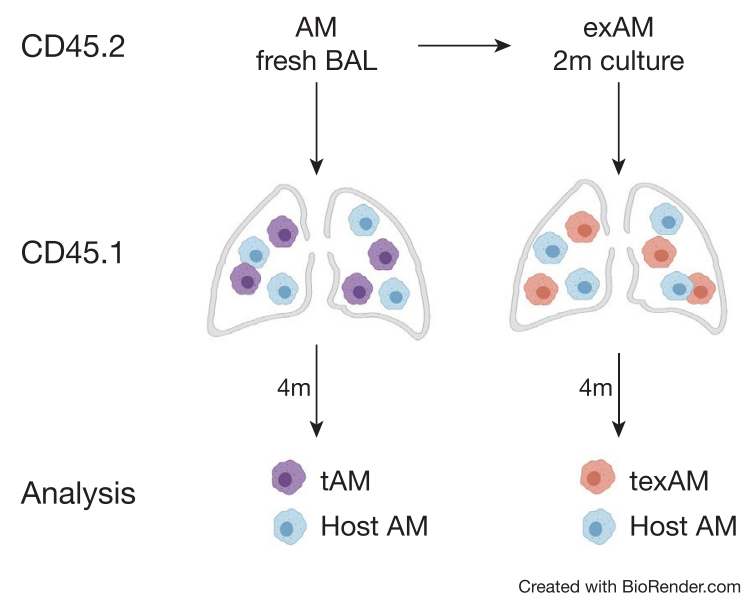

b

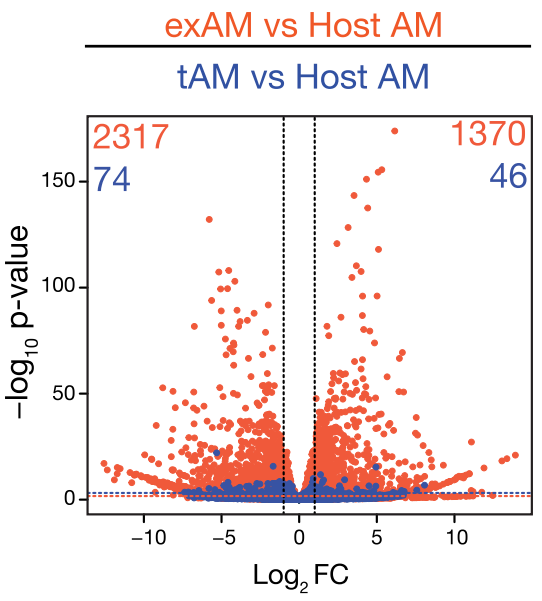

Extended Data Fig. 5 | Similar number of differences detected in tAM vs Host AM compared to exAM vs Host AM. (a) Experimental setup for intra-tracheal transplantation of BAL AM or 2 months expanded exAM (wild type, WT CD45.2) into lungs of WT CD45.1 host mice. Created with Biorender.com. (b) Overlaid volcano plot showing DEGs in exAM (red) or tAM (blue) compared to Host AM. Number of genes in blue and red with threshold $\mathrm{FC}>2$ and $\mathrm{FDR}<0.05$. 
Host AM
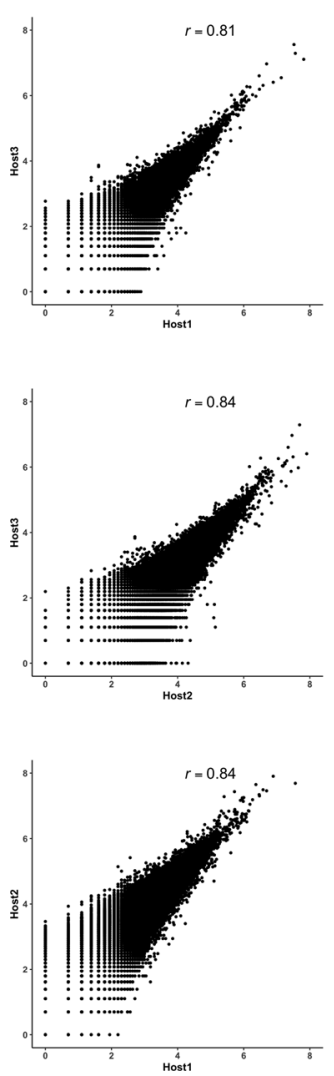

BAL AM
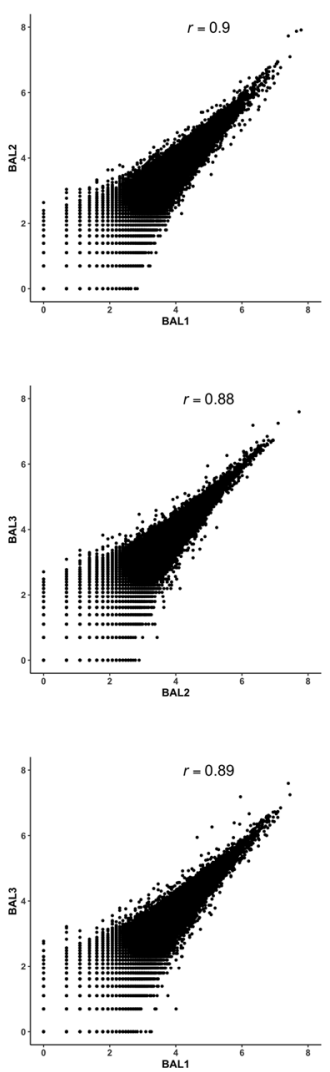

exAM
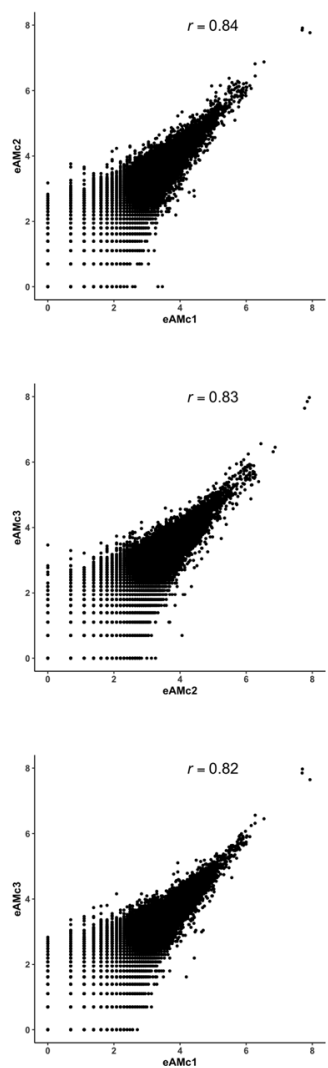

texAM
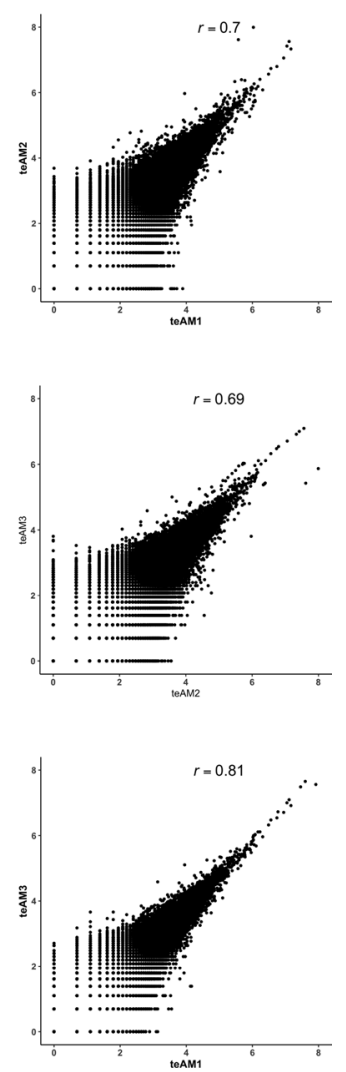

Extended Data Fig. 6 | Pearson correlation of ATAC-seq samples. Pearson correlation scatter-plots of replicates from pools of 3 mice for BAL AM, Host AM, exAM and texAM ATAC-seq samples. 


\section{TECHNICAL REPORT}

NATURE IMMUNOLOGY
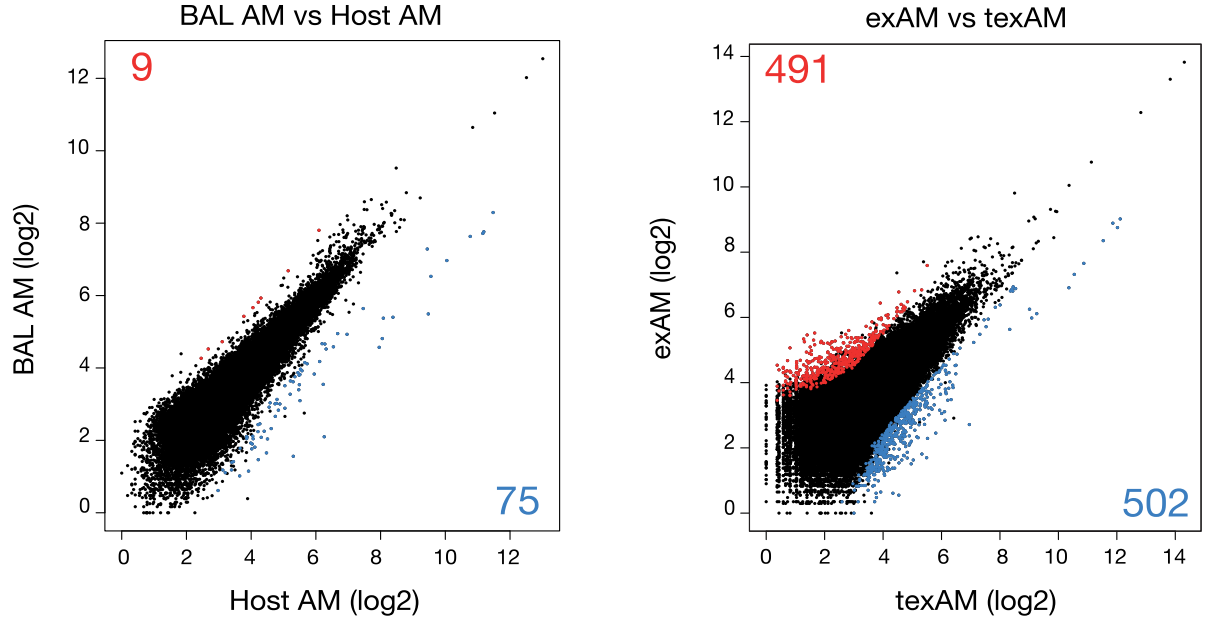

Extended Data Fig. 7 | Differential OCRs in BAL AM vs Host AM and in exAM vs texAM. Scatter-plot analysis of ATAC-seq peaks differentially gained (red) or lost (blue) in BAL AM vs Host AM and exAM vs texAM. 


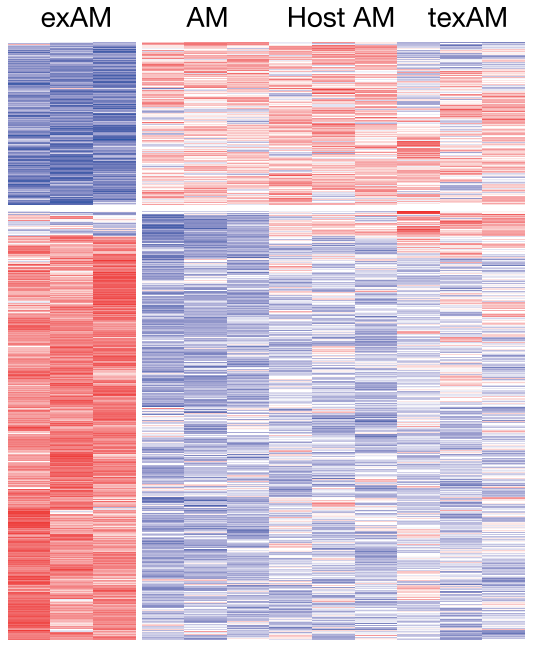

Extended Data Fig. 8 | Similar chromatin accessibility within in vivo AMs in contrast to exAM. Heat map of unsupervised clustering of differential ATAC-seq peaks across all conditions, showing rlog normalized values from minimum (blue) to maximum (red). 
exAM vs Host AM gained peaks

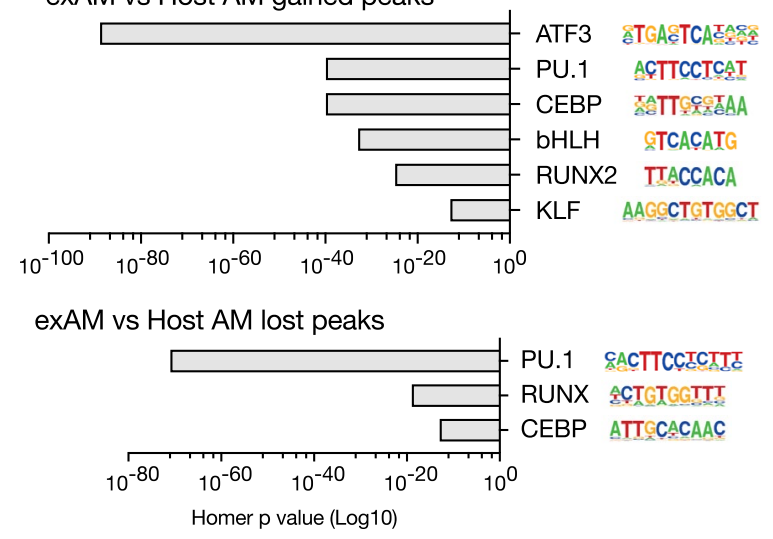

Extended Data Fig. 9 | Association of core myeloid transcription factors with gained and lost exAM OCRs. Motif enrichment analysis (MEA) for transcription factor (TF) binding sites on open chromatin region (OCR) gained (top panel) or lost (bottom panel) in exAM compared to host AM. 

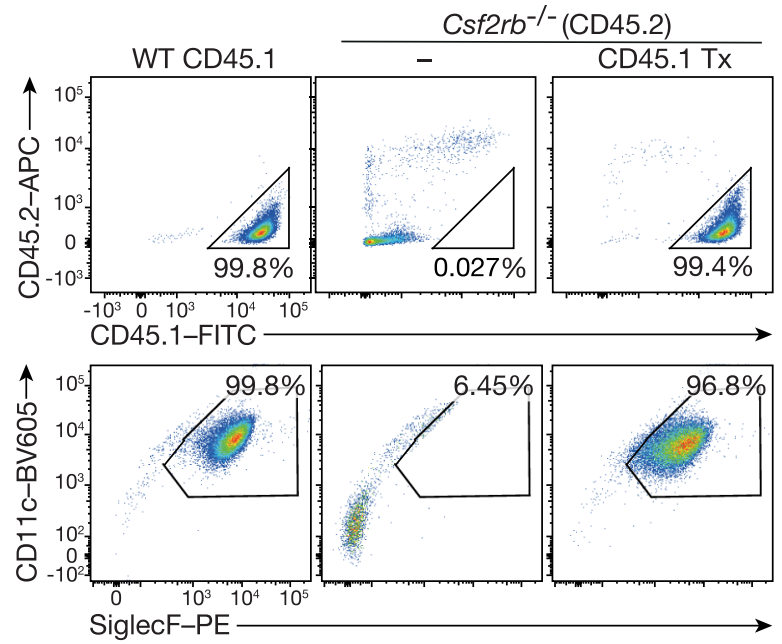

Extended Data Fig. 10 | exAM reconstitution of $C s f 2 \mathbf{r b}^{-\%}$ lungs at 3 months post transplantation. Flow cytometric analysis of exAM contribution to

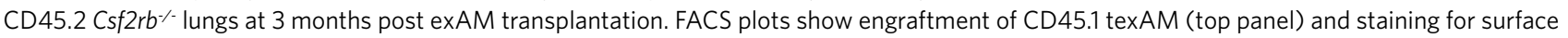
markers SiglecF and CD11c (bottom panel). Quantifications shown in Fig. 6b. 


\section{nature portfolio}

\section{Reporting Summary}

Nature Portfolio wishes to improve the reproducibility of the work that we publish. This form provides structure for consistency and transparency in reporting. For further information on Nature Portfolio policies, see our Editorial Policies and the Editorial Policy Checklist.

\section{Statistics}

For all statistical analyses, confirm that the following items are present in the figure legend, table legend, main text, or Methods section.

$\mathrm{n} / \mathrm{a}$ Confirmed

$\bigotimes$ The exact sample size $(n)$ for each experimental group/condition, given as a discrete number and unit of measurement

\A statement on whether measurements were taken from distinct samples or whether the same sample was measured repeatedly

The statistical test(s) used AND whether they are one- or two-sided

Only common tests should be described solely by name; describe more complex techniques in the Methods section.

Х A description of all covariates tested

Х $\square$ A description of any assumptions or corrections, such as tests of normality and adjustment for multiple comparisons

A full description of the statistical parameters including central tendency (e.g. means) or other basic estimates (e.g. regression coefficient) AND variation (e.g. standard deviation) or associated estimates of uncertainty (e.g. confidence intervals)

For null hypothesis testing, the test statistic (e.g. $F, t, r$ ) with confidence intervals, effect sizes, degrees of freedom and $P$ value noted Give $P$ values as exact values whenever suitable.

Х $\square$ For Bayesian analysis, information on the choice of priors and Markov chain Monte Carlo settings

Х $\square$ For hierarchical and complex designs, identification of the appropriate level for tests and full reporting of outcomes

$\square$ Estimates of effect sizes (e.g. Cohen's $d$, Pearson's $r$ ), indicating how they were calculated

\section{Our web collection on statistics for biologists contains articles on many of the points above.}

\section{Software and code}

Policy information about availability of computer code

Data collection FACSCanto, FACS LSRII and LSRFortessa and FACSArialll systems (BD bioscience).

Zeiss Axio Vert A1 microscope

Inverted microscope (Dmi1, Leica)

Zeiss inverse ApoTome microscope

Cytospin 4 Thermo Fisher

2100 Bioanalyzer (Agilent)

NextSeq 500 instrument (Illumina)

Data analysis Graphpad Prism version 9.0

FlowJo version 10 (Treestar)

Qupath version 0.2.0

FIJI version $1.52 \mathrm{n}$

Integrative genomics viewer (IGV) version 2.10 .2

Great tool version 4.0.4

Bedtools version 2.26.0

R package version 3.3

Bowtie version 1.1.2

Htseq-count version 0.6.1

EdgeR version 3.22.3

DESeq2 version 1.20

ChIPseeker version 1.26

Cutadapt version 2.6

Fastqc version 0.11.6

Genrich version 0.6.1 


\section{Data}

Policy information about availability of data

All manuscripts must include a data availability statement. This statement should provide the following information, where applicable:

- Accession codes, unique identifiers, or web links for publicly available datasets

- A description of any restrictions on data availability

- For clinical datasets or third party data, please ensure that the statement adheres to our policy

The sequencing data has been deposited in a public repository and the GEO accession codes will be made available upon publication.

\section{Field-specific reporting}

Please select the one below that is the best fit for your research. If you are not sure, read the appropriate sections before making your selection.

\ Life sciences

Behavioural \& social sciences

Ecological, evolutionary \& environmental sciences

For a reference copy of the document with all sections, see nature.com/documents/nr-reporting-summary-flat.pdf

\section{Life sciences study design}

All studies must disclose on these points even when the disclosure is negative.

Sample size

No sample size calculation was performed. All our in vitro and in vivo experiments contain at least $n>3$ biological replicates or $n>3$ technical replicates where each replicate was obtained from at least $n>2$ pooled biological samples. The number of technical or biological replicates and the number of experimental repeats are described in the methods section and in the figure legends in more detail.

Data exclusions Non-transplanted mice were excluded from analysis in Fig 6.

Replication Each experiment was repeated at least once and the number of repetitions are mentioned in each figure legend.

Randomization The mice were allocated randomly.

Blinding The data collection was not performed in a blinded manner since subjective measurement was not involved. We have chosen objective analysis and measurements in all our experiments and provided quantitative results. Library preparation and sequencing of the samples for RNAseq and ATACseq were performed in a blinded manner.

\section{Reporting for specific materials, systems and methods}

We require information from authors about some types of materials, experimental systems and methods used in many studies. Here, indicate whether each material, system or method listed is relevant to your study. If you are not sure if a list item applies to your research, read the appropriate section before selecting a response.

\begin{tabular}{l|l} 
Materials \& experimental systems \\
\hline $\mathrm{n} / \mathrm{a}$ & Involved in the study \\
$\square$ & $\bigotimes$ Antibodies \\
$\square$ Eukaryotic cell lines \\
$\square$ Palaeontology and archaeology \\
$\square$ & $\square$ Animals and other organisms \\
$\square$ & $\square$ Clinical data \\
$\square$ & $\square$ Dual use research of concern
\end{tabular}

Antibodies

Antibodies used

anti-CD11b (clone M1/70, eBioscience Cat\# 12-0112-82 1:500 or BD Cat\# 557657 1:400) 


\section{Eukaryotic cell lines}

Policy information about cell lines

Cell line source(s)

GM-CSF producing J558L cell line was kindly provided by Philippe Pierre's Lab at the CIML, Marseille, France.

\section{Authentication}

Mycoplasma contamination

Commonly misidentified lines

(See ICLAC register)
GM-CSF producing J558L cell line has been authenticated by verifying GM-CSF secretion in the supernatant by ELISA.

Cell lines and primary cells maintained in culture were tested and determined to be negative for mycoplasma.

No commonly misidentified cell lines were used in this study.

\section{Animals and other organisms}

Policy information about studies involving animals; ARRIVE guidelines recommended for reporting animal research

Laboratory animals

Wild animals

Field-collected samples

Ethics oversight

\section{Animals used were 2 to 4 months old.}

WT C57BL6/J CD45.1+ and CD45.2+ mice from Charles River and Janvier respectively were used.

Csf2rb-/- mice were used (Robb L et al., Proc Natl Acad Sci USA 1995).

Mice of 2-4 months of age were used in all experiments. In transplantation experiments, the age of donor and recipient mice were between 6 and 8 weeks old. Mice were housed under specific pathogen-free conditions maintaining temperatures of $21^{\circ} \mathrm{C}$ with $50 \%$ humidity in individually ventilated cages in a controlled 12-hour reverse light/dark cycle and were provided with food and water ad libitum. Only female mice were used for both RNA-seq and ATAC-seq experiments. For neonatal transplants, animals of both sexes were used. Alveolar macrophages isolated from both male and female mice were used in all other assays.

This study did not involve wild animals.

This study did not involve samples collected from the field.

All animal work was approved by the ethics committee of n014, Marseille, France (APAFIS\#3292-2015122109359224) and the TU Dresden and the Landesdirektion Sachsen in accordance with institutional, national and European animal welfare legislation (TVV28/2018 AKZ: IC 114-G0160/16 and DD24.1-5131/449/30).

Note that full information on the approval of the study protocol must also be provided in the manuscript.

\section{Flow Cytometry}

\section{Plots}

Confirm that:

\The axis labels state the marker and fluorochrome used (e.g. CD4-FITC).

$\bigotimes$ The axis scales are clearly visible. Include numbers along axes only for bottom left plot of group (a 'group' is an analysis of identical markers).

\All plots are contour plots with outliers or pseudocolor plots.

$\bigotimes$ A numerical value for number of cells or percentage (with statistics) is provided.

\section{Methodology}

Sample preparation

Mice were euthanized by cervical dislocation, lungs were collected, cut in small pieces and incubated with $1 \mathrm{mg} / \mathrm{ml}$ collagenase-2 (CLS2, Worthington) and $0.15 \mathrm{mg} / \mathrm{ml}$ DNasel (DN25, Sigma) at $37^{\circ} \mathrm{C}$ for $30 \mathrm{~min}$ with constant agitation. Cell suspension was filtered through a $70 \mu \mathrm{m}$ mesh and erythrocytes were removed by RBC lysis (RBC Lysis buffer, Cat\# 00-4333-57 Invitrogen). 
Software

Cell population abundance

Gating strategy
BD FACSDiva software was used for acquisition and sorting.

FlowJo software v10 (Treestar) was used for the cytometry analysis.

$\%$ of SiglecF+ CD11c+ alveolar macrophages sorted for RNAseq:

tAM1: 9.5\%; tAM2: 6.8\%; texAM1: 7.5\%; texAM2: 5.3\%; HostAM1: 88.3\%; HostAM2: 89.7\%

$\%$ of SiglecF+ CD11c+ alveolar macrophages sorted for ATAC-seq:

texAM1: 0.71\%; texAM2: 0.27\% ; texAM3: 12\% ; HostAM1: 98.8\% ; HostAM2: 99\%; HostAM3: $87.4 \%$

The purity of the sorted fractions were not determined.

We first used FSC/SSC to gate on cells. Dead cells were removed (Positive for the Fixable live/dead dye as indicated). Live cells were then gated depending on the analysis performed. (a) surface marker analysis: singlets gated and analyzed surface markers. (b) engraftment efficiency and sorting: Singlets, SiglecF+CD11c+ AM, CD45.1+/CD45.2+ cells were sorted.

$\bigotimes$ Tick this box to confirm that a figure exemplifying the gating strategy is provided in the Supplementary Information. 